\title{
Motif mimetic of epsin perturbs tumor growth and metastasis
}

\author{
Yunzhou Dong, ${ }^{1}$ Hao Wu, ${ }^{1}$ H.N. Ashiqur Rahman, ${ }^{1}$ Yanjun Liu, ${ }^{2}$ Satish Pasula, ${ }^{1}$ Kandice L. Tessneer, ${ }^{1}$ Xiaofeng Cai, ${ }^{1}$ Xiaolei Liu, ${ }^{1,3}$ \\ Baojun Chang, ${ }^{1}$ John McManus, ${ }^{1}$ Scott Hahn, ${ }^{1}$ Jiali Dong, ${ }^{1}$ Megan L. Brophy, ${ }^{1,3}$ Lili Yu, ${ }^{1}$ Kai Song, ${ }^{1}$ Robert Silasi-Mansat, \\ Debra Saunders, ${ }^{4}$ Charity Njoku, ${ }^{4}$ Hoogeun Song, ${ }^{1}$ Padmaja Mehta-D'Souza, ${ }^{1}$ Rheal Towner, ${ }^{4}$ Florea Lupu, ${ }^{1}$ Rodger P. McEver, ${ }^{1,3}$ \\ Lijun Xia, ${ }^{1,3}$ Derek Boerboom, ${ }^{5}$ R. Sathish Srinivasan, ${ }^{1}$ and Hong Chen ${ }^{1,3}$ \\ 'Cardiovascular Biology Program, Oklahoma Medical Research Foundation (OMRF), Oklahoma City, Oklahoma, USA. ${ }^{2}$ Charles R. Drew University of Medicine and Sciences, UCLA, Los Angeles, California, USA. \\ ${ }^{3}$ Department of Biochemistry and Molecular Biology, University of Oklahoma Health Sciences Center, Oklahoma City, Oklahoma, USA. ${ }^{4}$ Advanced Magnetic Resonance Center, OMRF, Oklahoma City, \\ Oklahoma, USA. ${ }^{5}$ Département de Biomédecine Vétérinaire, Université de Montréal, Montreal, Quebec, Canada.
}

\begin{abstract}
Tumor angiogenesis is critical for cancer progression. In multiple murine models, endothelium-specific epsin deficiency abrogates tumor progression by shifting the balance of VEGFR2 signaling toward uncontrolled tumor angiogenesis, resulting in dysfunctional tumor vasculature. Here, we designed a tumor endothelium-targeting chimeric peptide (UPI) for the purpose of inhibiting endogenous tumor endothelial epsins by competitively binding activated VECFR2. We determined that the UPI peptide specifically targets tumor endothelial VEGFR2 through an unconventional binding mechanism that is driven by unique residues present only in the epsin ubiquitin-interacting motif (UIM) and the VEGFR2 kinase domain. In murine models of neoangiogenesis, UPI peptide increased VECF-driven angiogenesis and neovascularization but spared quiescent vascular beds. Further, in tumor-bearing mice, UPI peptide markedly impaired functional tumor angiogenesis, tumor growth, and metastasis, resulting in a notable increase in survival. Coadministration of UPI peptide with cytotoxic chemotherapeutics further sustained tumor inhibition. Equipped with localized tumor endothelium-specific targeting, our UPI peptide provides potential for an effective and alternative cancer therapy.
\end{abstract}

\section{Introduction}

Angiogenesis, essential for embryogenesis and postnatal tissue repair, is often exploited by tumors to support accelerated growth and exaggerated cancer cell proliferation (1-7). The VEGF signaling critical for angiogenesis has been therapeutically modulated to curb tumor expansion $(3,5,8-11)$. Indeed, anti-VEGF therapies, such as Avastin (bevacizumab) (12), a humanized monoclonal antiVEGF-A Ab, have proven effective in the treatment of a wide variety of cancers (12-15). However, the rapid rate at which cancer cells mutate and adapt to changing microenvironments, including their dependence on tumor angiogenesis, highlights a continued need for new therapeutic strategies to combat the development of drug resistance in cancer (16). A better understanding of the molecular events governing tumor angiogenesis will therefore lead to alternative antiangiogenic strategies to complement current anticarcinogenic and antimetastatic treatments. One such approach would be to shift the balance of VEGFR2 signaling toward uncontrolled angiogenesis and dysfunctional tumor vasculature $(17,18)$. Identifying and designing therapeutics that modulate these alternative events will significantly advance cancer therapy.

Endocytosis is an important process involved in the modulation of numerous cell-signaling pathways (19). Altered endocytic regulation of various monoubiquitinated cell surface receptors

Conflict of interest: The authors have declared that no conflict of interest exists. Submitted: December 5, 2014; Accepted: August 6, 2015.

Reference information: / Clin Invest. 2015;125(12):4349-4364. doi:10.1172/JCI80349. has been linked to pathological cancer progression; therefore, targeted endocytic manipulation has been implicated as a potential strategy for future cancer therapy (20). Epsins are a family of endocytic clathrin adaptors and ubiquitin-binding proteins (21$23)$ with emerging importance in human disease $(24,25)$. Despite abnormally high epsin expression in tumor tissues $(24,25)$, its procancer role remains elusive. Global deletion of both epsins 1 and 2 is embryonically lethal in mice (26), but postnatal endothelial cell-specific deletion of epsins produces adult mice with no gross physiological defects in quiescent vessels (17). These mice did, however, exhibit altered tumor angiogenesis consisting of highly disorganized, nonproductive and hyperpermeable tumor vasculature that resulted in tumor growth inhibition (17). The vascular dysfunction responsible for the profound tumor-resistant phenotype was a result of impaired VEGFR2 internalization and degradation and resulted in failed downregulation of VEGF signaling $(17,18)$. VEGF stimulates VEGFR2 internalization and degradation in part by inducing VEGFR2 ubiquitination (17). We determined that epsins, via their ubiquitin-interacting motif (UIM), recognize ubiquitinated VEGFR2 at the plasma membrane and recruit it to clathrin-coated pits for internalization and subsequent degradation, thereby reducing VEGF signaling $(17,18)$. In this capacity, epsin loss exacerbated VEGF signaling, disrupted tumor angiogenesis, and inhibited tumor progression (17). Our counterintuitive finding that exacerbated tumor angiogenesis can actually inhibit tumors prompted us to search for a new strategy to combat human cancer by specifically disrupting 
epsin-mediated downregulation of VEGFR2 signaling in tumor endothelium. Given that the epsin UIM sequence is highly conserved among human and mouse epsins 1 and 2 (Figure 1A) and that the epsin UIM is critical for epsin-VEGFR2 binding (17), we predict that the epsin UIM may be a central element in epsin function and, therefore, a potential target for clinical applications.

\section{Results}

Tumor-targeting epsin inhibitory peptide homes to and impairs epsinVEGFR2 interactions within tumor vascular endothelial cells. We postulated that a synthetic UIM peptide designed to competitively prohibit epsin from binding to ubiquitinated VEGFR2 could disrupt functional tumor angiogenesis and thereby tumor growth (17). Toward this end, we chemically synthesized a UIM-containing, tumor vasculature-targeting peptide, referred to herein as UPI (Figure 1B and Supplemental Table 1; supplemental material available online with this article; doi:10.1172/JCI80349DS1). Specifically, we conjugated the C-terminus of our UIM peptide to the tumor endothelial cell-homing (TEC-homing) peptide iRGD, which facilitates peptide homing to and internalization by TECs $(27,28)$. iRGD mediates the specific uptake of iRGD-conjugated peptides by TECs through first binding $\alpha v \beta 3$ or $\alpha v \beta 5$ integrin, then neuropilin 1, which is overexpressed on the surface of TECs (28). To further enrich plasma membrane localization of the UIM peptide conjugate, a plasma membrane-anchoring peptide from the Lyn kinase H4 domain $(22,29)$, known to bind lipid rafts through palmitoylation and myristoylation, was inserted between the UIM and iRGD. Molecular modeling revealed that this UPI peptide forms a helical structure (Figure 1C) and, consistent with epsin UIM function, binds ubiquitin in a manner similar to that observed in the UIM of yeast Vps27 (Figure 1D and reviewed in ref. 30). Molecular modeling further predicted that the UPI peptide can directly interact with the cytoplasmic domain of VEGFR2, providing an important rationale for its potential competitive inhibition of epsin binding to VEGFR2 (Figure 1E).

To evaluate the targeting specificity and efficacy of our UPI, we treated human umbilical vein endothelial cells (HUVECs) overexpressing $\alpha v \beta 3$ integrin (HUVEC ${ }^{\alpha v \beta 3}$ ) with FITC-conjugated UPI (FUPI) (Supplemental Table 1). Normal quiescent endothelial cells, such as HUVECs, express endogenous $\alpha v \beta 3$ integrin. However, compared with TECs, this expression is considerably low. By overexpressing $\alpha \mathrm{v} \beta 3$ integrin in HUVECs, we distinguished the ability of the peptide to target endothelial cells in quiescent versus tumor vasculature. Overexpression of $\alpha v \beta 3$ integrin was monitored by FACS and Western blotting (Figure $1 \mathrm{~F}$ and Supplemental Figure 1A). As predicted, FUPI peptide accumulated selectively in HUVEC ${ }^{\alpha v \beta 3}$, but not in HUVECs alone (Figure 1G). Examination of the intracellular kinetic stability of FUPI peptide in HUVEC ${ }^{\alpha v \beta 3}$ revealed maximal intracellular accumulation 15-24 hours after administration (Supplemental Figure 1, B and C). Further, FUPI colocalized with VEGFR2 at the plasma membrane of HUVEC ${ }^{\alpha v \beta 3}$, presumably because of the inclusion of the plasma membrane-anchoring sequence (Supplemental Figure 1D). To validate its tumor vasculaturetargeting efficacy and stability in vivo, FUPI was administered i.v. to Lewis lung carcinoma (LLC) and B16F10 (B16) melanoma s.c. tumor-bearing mice. To circumvent stability issues com- mon in i.v. administered peptide therapies, we designed a cyclic iRGD-homing sequence within the UPI (31). FUPI peptide was detected within CD31-positive intratumoral vessels as early as 1 hour and was retained for up to 48 hours after administration (Figure $1 \mathrm{H}$ and Supplemental Figure 1E). Importantly, FUPI peptide was concentrated within tumor vasculature, but not in other major organs including liver, lung, heart, spleen, kidney, colon, and brain (Figure 1I and Supplemental Figure 2A). These findings strongly suggest that, through incorporation of the iRGD and plasma membrane-anchoring sequences, the UPI peptide effectively and specifically homes to the plasma membrane of tumor vascular endothelial cells.

We previously established that endothelial epsins specifically interact with and modulate tumor angiogenesis by facilitating the internalization and downregulation of ubiquitinated VEGFR2 $(17,18)$. Further, we determined that the epsin-VEGFR2 interaction was mediated by the epsin UIM. To ensure that our epsin UIM mimetic peptide UPI selectively impaired the epsinVEGFR2 interaction, we examined the competitive binding of UPI peptide in VEGF-stimulated 293T cells overexpressing VEGFR2 kinase domain (KD) and epsin 1. Cell lysates were incubated with control or UPI peptides (Supplemental Table 1), followed by co-IP analysis of VEGFR2 KD and epsin 1. UPI, but not control peptides, substantially abolished epsin 1 interaction with VEGFR2 KD (Figure 1J). Additionally, UPI peptide treatment effectively inhibited endogenous epsin 1-VEGFR2 binding when administered to intact HUVEC ${ }^{\alpha \vee \beta 3}$ and analyzed by co-IP assay of epsin 1 by VEGFR2 (Supplemental Figure 1F). Endothelial epsin deficiency markedly hindered VEGFR2 internalization, resulting in elevated VEGFR2 cell surface expression (17). FACS analysis of cell surface VEGFR2 expression and cell surface biotin-labeled VEGF internalization assays in UPI peptide-treated HUVEC $^{\alpha \nu \beta 3}$ revealed impaired endocytosis of VEGFR2 and substantial increases in VEGFR2 accumulation at the plasma membrane (Supplemental Figure 1, G-I). Collectively, these findings suggest that the UPI peptide successfully competes with epsin function and, in endothelial cells, is sufficient to impair epsinmediated VEGFR2 internalization.

Tumor vessel-targeting UPI peptide effectively impairs tumor growth and metastasis. To investigate the therapeutic potential of our tumor vessel-targeting UPI peptide, LLC s.c. tumor-bearing mice with established tumors of $20-50 \mathrm{~mm}^{3}$ in size were injected i.v. with UPI peptide on alternating days for 14 days using concentrations of 2.5, 5, and $10 \mathrm{mg} / \mathrm{kg}$. Control peptide was i.v. injected at $10 \mathrm{mg} / \mathrm{kg}$. LLC tumor growth, monitored on alternating days during peptide administration, was significantly impeded by UPI peptide in a dose-dependent manner, with $10 \mathrm{mg} / \mathrm{kg}$ yielding maximal inhibition (Figure 2A). Similarly, UPI peptide administered i.v. at $10 \mathrm{mg} / \mathrm{kg}$ every other day significantly retarded s.c. B16 melanoma tumor growth (Figure 2B) and s.c. human U87/SCID tumor growth (Figure 2C). To evaluate the therapeutic potential of our UPI peptide in a spontaneous tumor model, we used transgenic adenocarcinoma of mouse prostate (TRAMP) mice, which develop progressive prostate cancer between 10 and 20 weeks of age with $100 \%$ frequency (32). We administered $20 \mathrm{mg} / \mathrm{kg}$ UPI peptide by i.p. injection into mice on alternating days starting at 20 weeks of age to ensure tumor presence prior to peptide delivery. Mice were 
A

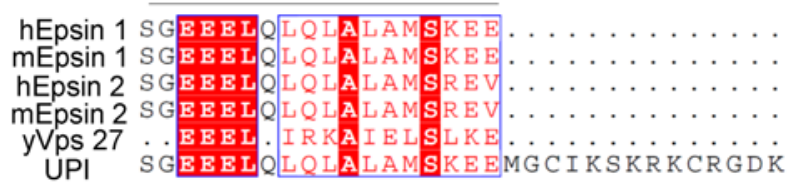

C
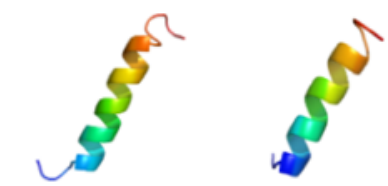

Yeast Vps27 UIM Epsin UIM

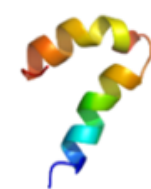

UPI peptide

B

\begin{tabular}{|c|c|c|}
\hline \multicolumn{1}{c|}{ UIM } & PM anchor & iRGD \\
\hline SGEEELQLQLALAMSKEE & MGCIKSKRK & CRGDKGPDC \\
\hline
\end{tabular}
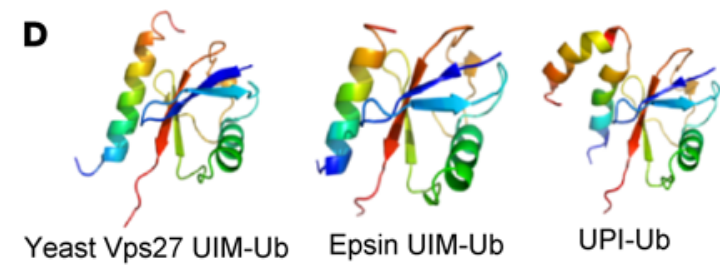

Epsin UIM-Ub

UPI-Ub

E

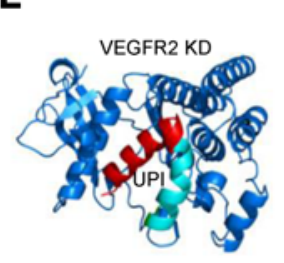

$\mathbf{F}$

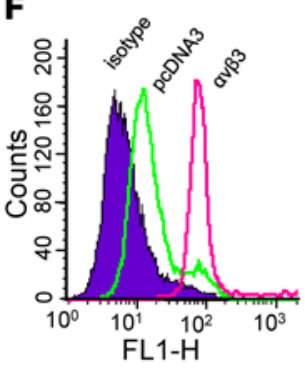

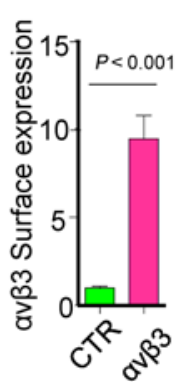

G

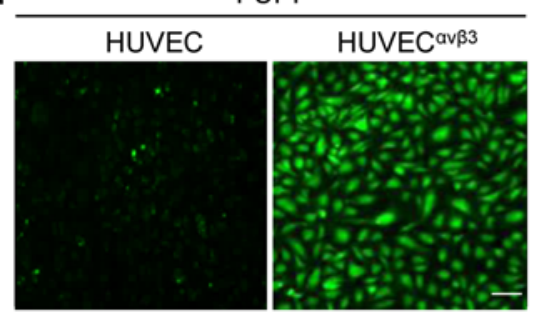

H

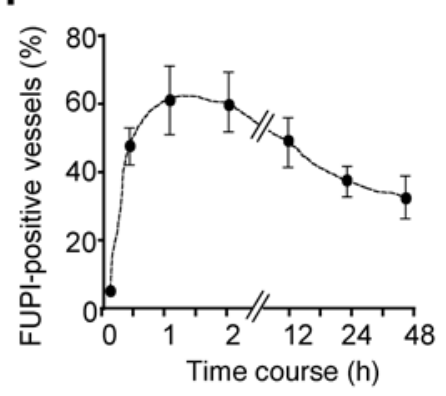

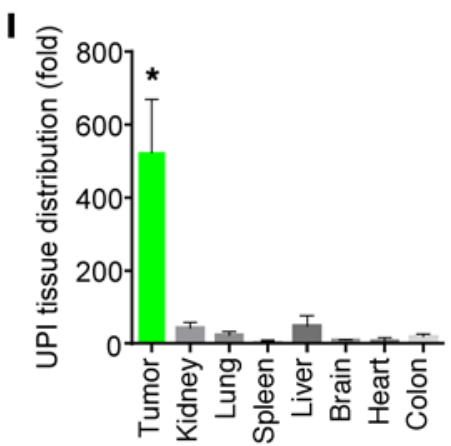

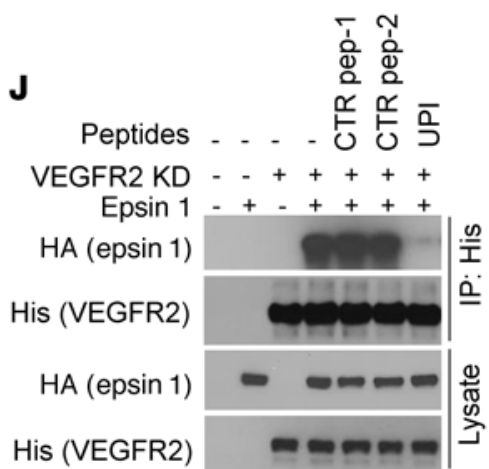

Figure 1. UPI mimetic targets endothelial cells and tumor vasculature to block epsin-VEGFR2 interaction. (A) Alignment of the UPI mimetic peptide with UIMs of human epsins (hEpsin), murine epsins (mEpsin), and yeast Vps27 (yVps). (B) Schematic of the UPI peptide depicting the UIM (pink), plasma membrane-anchoring (PM anchor) sequence (green), and iRGD-homing sequence (yellow). (C) Ribbon diagrams of yeast Vps27 UIM (left), epsin UIM (middle), and UPI (right) peptides predicted using PEP-FOLD. (D) Ribbon diagrams of yeast Vps27 UIM-ubiquitin (Ub) complex (left), human epsin UIM-Ub complex (middle), and UPI-Ub complex (right). (E) Ribbon diagram showing UPI peptide (red) docked into the VEGFR2 KD (blue). (F) FACS analysis of surface $\alpha v \beta 3$ expression in HUVECs transfected with HUVEC ${ }^{\alpha u \beta 3}$. HUVECs transfected with pcDNA3 were used as a control (see also Supplemental Figure $1 A$ ). $P<0.001$, by 2-tailed Student's $t$ test. (C) FUPI selectivity in HUVECs and HUVEC ${ }^{\alpha u \beta}$ treated with $10 \mu M$ FUPI for 15 hours. Representative image $(n=5)$. Scale bar: 100 $\mu \mathrm{m}$. (H) Pharmacokinetic analysis of FUPI peptide stability in tumor vasculature of s.c. B16 tumor-bearing mice injected i.v. once with FUPI peptide (10 mg/ $\mathrm{kg})$. Tumors were harvested at the indicated time points and processed for immunofluorescence staining with anti-CD31 Ab. Data represent the average percentage of FUPI peptide-positive vessels relative to total CD31-positive vessels $(n=5)$. See also Supplemental Figure 1E. (I) Tissue distribution of FUPI peptide in s.c. LLC tumor-bearing mice 4 hours after i.v. injection (10 mg/kg FUPI). FITC fluorescence intensity was analyzed by confocal microscopy and quantified $(n=4)$. ${ }^{*} P<0.001$, by 1-way ANOVA. See also Supplemental Figure 2A. (J) Representative Western blot showing UPI peptide inhibition of epsin 1 IP by VEGFR2 KD (His) analyzed by coincubating control or UPI peptide and anti-His Ab in lysates from 293T cells overexpressing HA-tagged epsin 1 and His-tagged VEGFR2 KD $(n=5)$. CTR, control; pep-1, peptide 1; pep-2, peptide 2.

sacrificed at 35 weeks and examined for primary prostate tumors. While large tumors were observed in the prostate and nearby seminal vesicles of control peptide-treated TRAMP mice, smaller and less frequent tumors were observed in mice that received UPI peptide (Figure 2D and Supplemental Figure 2B). These data strongly support the use of homing sequences to specifically target epsin UIM to tumor vascular endothelial cells as a potential therapeutic approach to combat tumor growth. Further, by using the plasma membrane-targeting sequences to effectively home the UPI peptide to the plasma membrane of TECs, we achieved significantly greater tumor inhibition relative to that of the UIM peptide conjugated to the nonselective plasma membrane-permeable peptide antennapedia (AP) (also known as penetratin) (AP-UIM) or to that of the UIM peptide containing only iRGD (UI peptide) (Sup- 
A

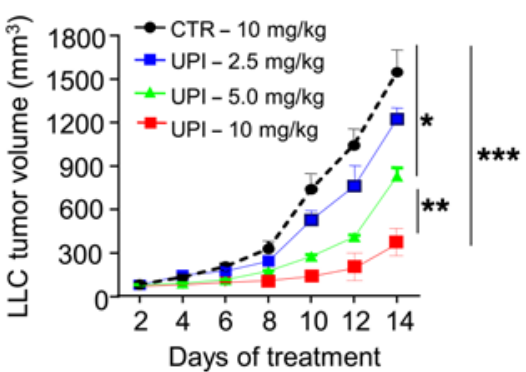

C

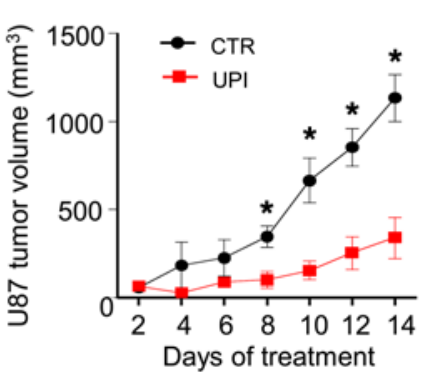

B

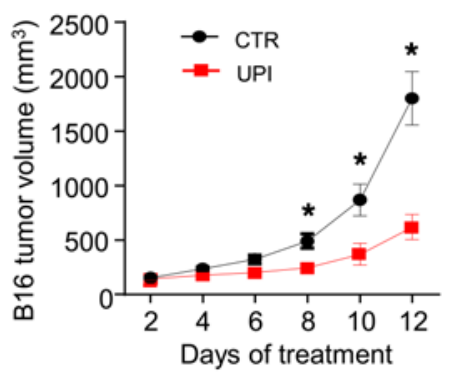

D

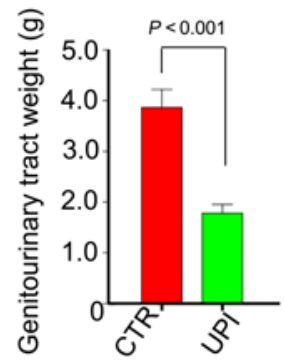

Figure 2. UPI mimetic inhibits tumor growth. (A) Tumor volumes from s.c. LLC tumor-bearing mice treated i.v. with UPI peptide $(2.5 \mathrm{mg} / \mathrm{kg}$, $n=8 ; 5 \mathrm{mg} / \mathrm{kg}, n=8$; and $10 \mathrm{mg} / \mathrm{kg}, n=10$ ) every other day. Control peptide was administered i.v. at $10 \mathrm{mg} / \mathrm{kg}(n=10) .{ }^{*} P<0.05$, ${ }^{* *} P<0.05$, ${ }^{* *} P<0.01$. (B) Tumor volumes from s.c. B16 tumor-bearing mice treated i.v. with $10 \mathrm{mg} / \mathrm{kg}$ UPI peptide $(n=10)$ every other day. Control peptide was administered i.v. at 10 $\mathrm{mg} / \mathrm{kg}(n=10) .{ }^{*} P<0.05$. (C) Tumor volumes from s.c. U87 glioma tumor-bearing immunodeficient SCID mice treated i.v. with $10 \mathrm{mg} / \mathrm{kg}$ UPI peptide $(n=10)$ every other day. Control peptide was administered i.v. at $10 \mathrm{mg} / \mathrm{kg}$ $(n=8)$. $(\mathbf{A}-\mathbf{C}){ }^{*} P<0.05$, by 2 -tailed Student's $t$ test and by Bonferroni's multiple comparisons test. (D) Genitourinary tracts were isolated from TRAMP mice treated i.p. with control or UPI peptides $(20 \mathrm{mg} / \mathrm{kg}$, every other day starting at week 20) and weighed $(n=15) . P<0.001$, by 2 -tailed Student's $t$ test. Right panel is a representative image. plemental Figure 2C and Supplemental Table 1), reinforcing the importance of plasma membrane microenvironment enrichment of the UIM peptide (33).

Given the specificity of our UPI peptide in targeting the tumor vasculature and the functional importance of tumor angiogenesis for establishing metastatic potential (34-36), we reasoned that our UPI peptide may impair metastasis by disrupting functional tumor angiogenesis. Because of the aggressive nature of the spontaneous TRAMP model, we monitored tumor metastasis in the UPI peptide-treated TRAMP mice. Gross morphological analysis as well as H\&E staining revealed dramatic reductions in the frequency of metastasis to the lungs and liver of UPI peptide-treated TRAMP mice (Figure 3, A and B). Accordingly, expression of the cancer cell marker vimentin and the metastatic marker snail was dramatically reduced in lung and liver tissue lysates isolated from UPI peptide-treated TRAMP mice (Figure 3C). Importantly, TRAMP mice receiving UPI peptide treatment had an average increased life expectancy of 3 months or longer(Figure 3D). However, smaller tumors metastasize less frequently, and since UPI peptide treatment reduced the primary tumor size in TRAMP mice, we cannot rule out the possibility that reduced metastasis is not a secondary result of impaired primary tumor growth. To address this, we used mice with highly metastatic s.c. B16 melanoma tumors. After primary B16 tumors reached 400 to $500 \mathrm{~mm}^{3}$ in size, they were surgically removed, then mice were randomized into 2 groups and postoperatively administered control or UPI peptide for 5 weeks. UPI peptide treatment significantly inhibited B16 melanoma metastasis to lymph nodes and lungs, as indicated by decreased vimentin and melan-a (specific melanoma marker) immunofluorescence staining (Figure 3, E and F), reduced the number of metastatic cancer cells in lymph nodes and the number of tumor nodules (foci) in lungs (Figure 3, E and F), and attenuated vimentin and snail protein levels in lungs (Figure $3 G$ ). Collectively, these data indicate that UPI peptide admin- istration not only impedes primary tumor growth but also significantly attenuates metastasis, presumably by disrupting functional tumor angiogenesis.

UPI peptide selectively disrupts epsin-VEGFR2 interaction and prevents epsin-mediated VEGFR2 downregulation in vitro and ex vivo. Molecular modeling (Figure 1E) and binding studies (Figure 1J and Supplemental Figure 1F) strongly suggest that the therapeutic efficacy of the UPI peptide stems from its competitive inhibition of epsin-VEGFR2 interaction. Consistent with these findings, UPI peptide-mediated inhibition of the epsin 1-VEGFR2 interaction augmented VEGF-induced VEGFR2 phosphorylation (Figure 4A) and increased phosphorylation of the downstream effectors PLC $\gamma$ and ERK in HUVEC ${ }^{\alpha v \beta 3}$ (Figure 4A). To examine whether this result is faithfully reproducible in TECs, we isolated and treated TECs from s.c. LLC tumors with VEGF in the presence or absence of UPI peptide. As shown in Figure 4B, UPI peptide treatment considerably augmented VEGFR2 signaling. To test the specificity of UPI peptide-mediated targeting of the VEGF signaling pathway, HUVEC $^{\alpha v \beta 3}$ were treated with a combination of control IgG or antiVEGF Ab and either UPI or control peptide prior to VEGF stimulation. Anti-VEGF Ab administration suppressed UPI peptide-mediated increases in VEGFR2 phosphorylation (Supplemental Figure 3, A and B). Collectively, these studies endorse the design of our UPI peptide as an inhibitor of epsin function that selectively targets VEGFR2 signaling in endothelial cells.

Multistep enrichment of the UPI peptide in TECs and sustained VEGF-dependent VEGFR2 signaling could prove critical for maximizing UPI peptide-mediated tumor inhibition through aberrant tumor angiogenesis. However, whether the UPI peptide targets other angiogenic signaling pathways in addition to VEGFR2 is unknown. To address this, we coincubated biotinylated UPI or control peptides and NeutriAvidin beads in lysates from LLC ex vivo tumors. Western blot analysis of pulldowns showed strong coprecipitation of VEGFR2 by UPI peptide (Figure 4C). However, UPI peptide failed to coprecipitate EGFR, FGFR1, or PDGFR- $\beta$ 
A

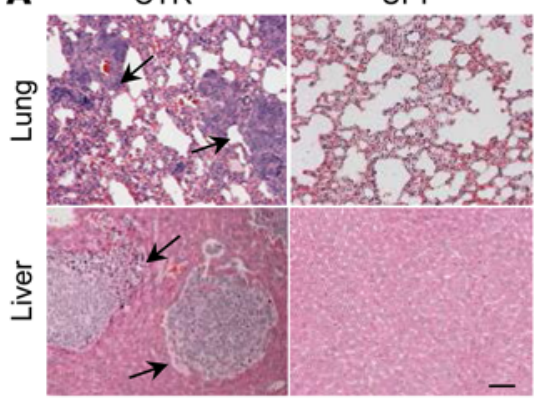

D

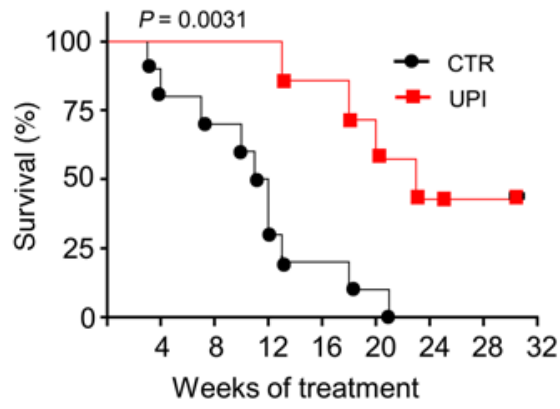

B

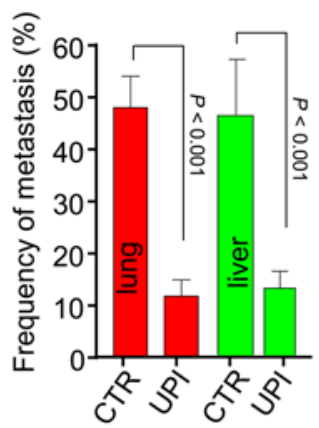

E
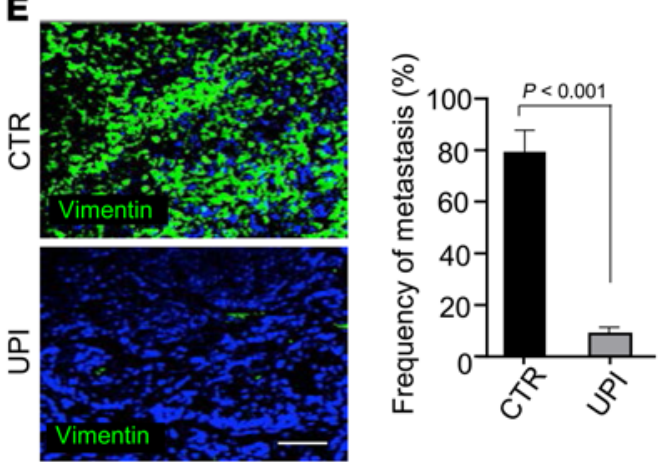

$\mathbf{F}$

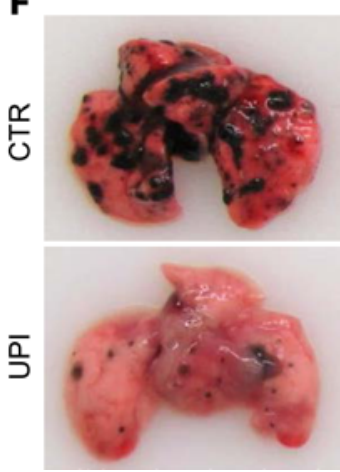

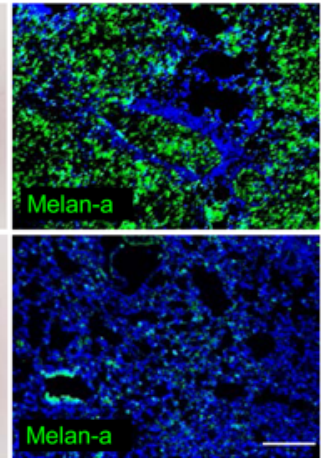

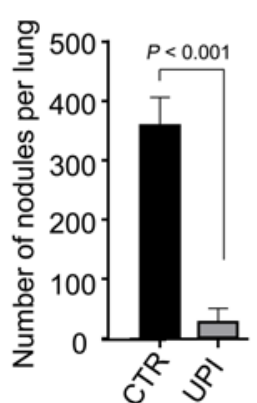

C

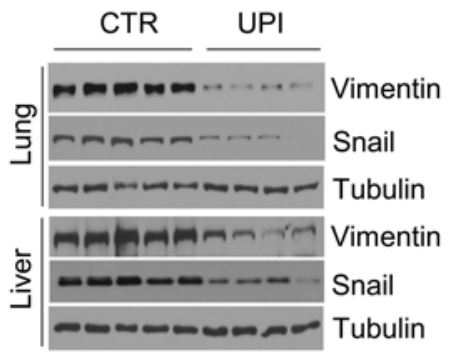

Figure 3. UPI mimetic inhibits metastasis and increases survival rates. (A) H\&E staining of lung and liver metastasis in TRAMP mice treated i.p. with control or UPI peptides (20 mg/kg, $n=15)$. Arrows indicate metastatic tumors. (B) Quantification of lung and liver metastasis $(n=15)$. $P<0.001$, by 2-tailed Student's $t$ test. (C) Western blot analysis of metastatic markers vimentin and snail in liver and lung tissue lysates isolated from TRAMP mice treated i.p. with control or UPI peptides ( $20 \mathrm{mg} / \mathrm{kg}$ ) ( $n>5$ for each group). (D) Survival plot of TRAMP mice after i.p. administration of control or UPI peptide (20 $\mathrm{mg} / \mathrm{kg}$ ) every other day starting at 20 weeks of age ( $n=20$ in the control group and $n=18$ in the UPI peptide-treated group). $P=0.0031$, by Mantel-Cox log-rank test. (E) Immunofluorescence analysis of the metastatic marker vimentin (green) in lymph nodes isolated from control and UPI peptide-treated s.c. B16 tumor-bearing mice 5 weeks after surgical removal of primary tumors. Metastasis frequency was quantified as the percentage of metastasis-positive lymph nodes $(n>20) . P<0.001$, by 2-tailed Student's $t$ test. $(\mathbf{F})$ Gross (left) and immunofluorescence (middle) analysis of lung metastasis in control and UPI peptide-treated B16 tumor-bearing mice 5 weeks after surgical removal of primary tumors. Immunofluorescence staining was done with the melanoma-specific marker melan-a. The number of metastatic nodules in each lung was quantified and depicted in a histogram (right) ( $n=10$ ). $P<0.001$, by by 2-tailed Student's $t$ test. (G) Representative Western blot of metastatic markers in lung tissues isolated from control and UPI peptide-treated B16 tumor-bearing mice 5 weeks after surgical removal of primary tumors $(n=5)$.

(Figure 4C), suggesting that the UPI peptide binds VEGFR2 specifically, a finding consistent with epsin specificity for VEGFR2 (17). This was further confirmed using biotinylated UPI peptide in an ELISA (Supplemental Figure 3C). To further investigate the targeting specificity of UPI peptide for VEGFR2, downstream signaling of FGF, PDGF, and EGF stimulation was assessed after UPI pep- tide treatment of HUVEC ${ }^{\alpha v \beta 3}$ (Figure 4D and Supplemental Figure 3, D-F). Consistently, these major angiogenic receptor signaling pathways were not affected by UPI peptide. Similarly, VEGFR2, but not other angiogenic receptors, was increased in tumors from UPI peptide-treated tumor-bearing mice (Supplemental Figure 3, G-I). UPI peptide did not alter endogenous epsin protein levels in 
A

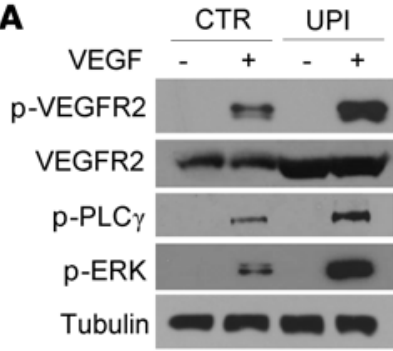

B

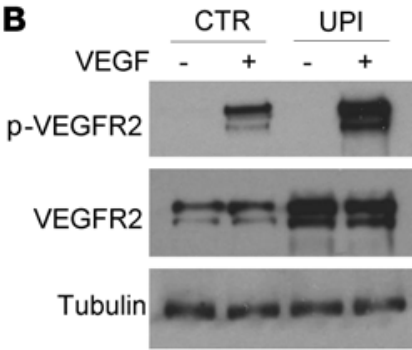

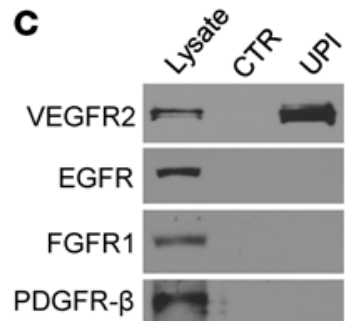

D

FGF $\frac{\text { CTR }}{+\cdots \cdots} \frac{\text { UPI }}{\cdots+\cdots}$

PDGF - - - + . . + +

EGF - - - . + . . - +

p-PLC $\gamma--\cdots-\cdots$
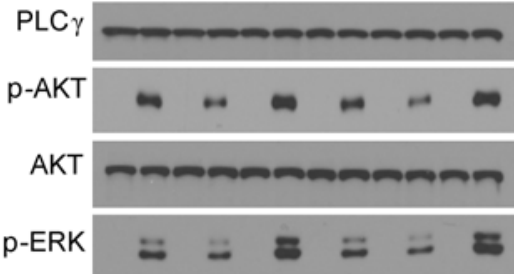

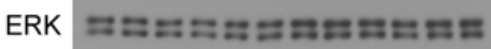

Tubulin -
E

\begin{tabular}{|c|c|c|c|c|c|c|c|c|c|c|}
\hline \multirow[b]{2}{*}{ Epsin 1} & & \multirow[b]{2}{*}{$\mathbf{S}$} & \multirow[b]{2}{*}{ GEEEL } & \multicolumn{2}{|c|}{$\begin{array}{c}\text { Q9 } \\
\downarrow\end{array}$} & \multicolumn{2}{|r|}{$\stackrel{\mathrm{A} 13}{\downarrow}$} & \multicolumn{2}{|c|}{$\begin{array}{c}\mathrm{K} 16 \\
\downarrow\end{array}$} & \\
\hline & & & & Q L & QL & A & LA & MS & $\mathbf{K}$ & EE \\
\hline Hrs-A & - & $\mathbf{Q}$ & EEEEL & QL & AL & $\mathbf{A}$ & LS & Q S & $\mathbf{E}$ & AE \\
\hline Hrs-B & . & $\mathbf{Q}$ & EEEEL & QL & AL & A & LS & Q S & $\mathbf{E}$ & AE \\
\hline Eps15-1 & . & $\mathbf{s}$ & EEDMI & EW & A.K & $\mathbf{R}$ & ES & E R & $\mathbf{E}$ & EE \\
\hline Eps15-2 & . & $\mathbf{Q}$ & EQEDL & EL & AI & A & LS & K S & $\mathbf{E}$ & IS \\
\hline Vps & - & D & EEELI & RK & AI & $\mathbf{E}$ & LS & L K & $\mathbf{E}$ & SR \\
\hline Vps27-2 & . & $\mathbf{E}$ & ED PDL & KA & AI & Q & ES & L R & $\mathbf{E}$ & $A E$ \\
\hline Stam1-A & & $\mathbf{K}$ & EEEDL & AK & AI & $\mathbf{E}$ & LS & L K & $\mathbf{E}$ & QR \\
\hline Stam1-B & & $\mathbf{K}$ & EEEDL & AK & AI & $\mathbf{E}$ & LS & L K & $\mathbf{E}$ & QR \\
\hline $\operatorname{tam} 2-A$ & & $\mathbf{K}$ & EDEDI & AK & AI & $\mathbf{E}$ & LS & L Q & $\mathbf{E}$ & QK \\
\hline tam2-B & . & $\mathbf{K}$ & EDEDI & AK & AI & $\mathbf{E}$ & LS & I Q & $\mathbf{E}$ & QK \\
\hline
\end{tabular}

$\mathbf{F}$
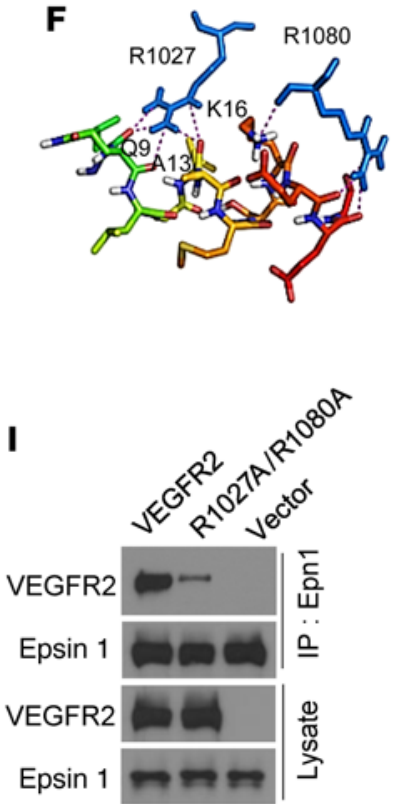

G
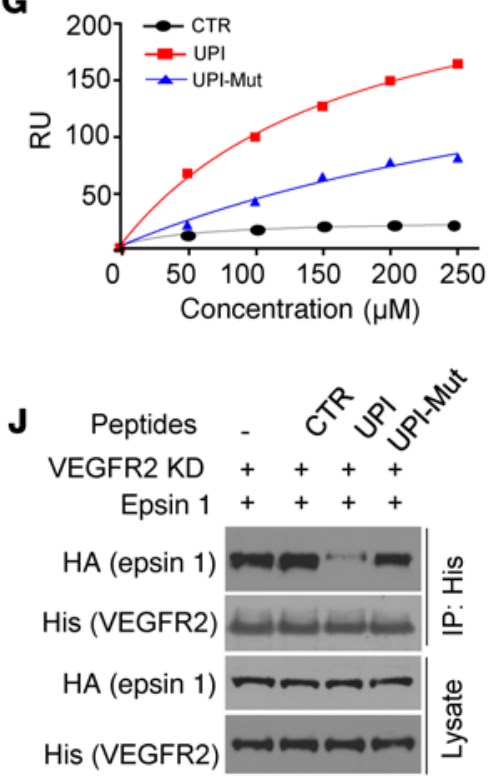

Figure 4. UPI mimetic specifically alters VEGFR2 signaling in vitro and ex vivo. (A) Western blot analysis of VEGF-mediated (50 ng/ml, $5 \mathrm{~min})$ VEGFR2 accumulation and phosphorylation and downstream phosphorylation of PLC $\gamma$ and ERK in HUVEC ${ }^{\alpha \nu \beta 3}$ pretreated with $10 \mu \mathrm{M}$ control or UPI peptide ( $n=5$ ). (B) Western blot analysis of total and VECF-mediated ( $50 \mathrm{ng} / \mathrm{ml}, 5 \mathrm{~min}$ ) p-VECFR2 in TECs pretreated with $10 \mu \mathrm{M}$ control or UPI peptide. TECs were isolated from LLC tumors $(n=5)$. (C) Western blot analysis of VEGFR2, EGFR, FGFR1, and PDGFR- $\beta$ precipitation by biotinylated control or UPI peptides in ex vivo LLC tumor lysates $(n=5)$. (D) Western blot analysis of FGF, PDGF, and EGF-mediated downstream phosphorylation of PLC $\gamma$, AKT, and ERK in HUVEC av 33 pretreated with $10 \mu M$ control or UPI peptide $(n=5)$. (E) Alignment of UIM sequences from various UIM-containing endocytic proteins. Note: Q9, A13, and K16 are residues uniquely present in the epsin 1 UIM. (F) Molecular diagram of the epsin UIM docking into the putative binding pocket of ubiquitinated VEGFR2 KD. (C) SPR analysis of CTR, UPI, or UPI mutant peptide binding to purified VEGFR2 KD. UPI mutant peptide has Q9A, A13S, and K16A amino acid exchanges $(n=4)$. RU, response units. (H) Western blot analysis of epsin 1 co-IP by VEGFR2 in 293T cell overexpression of VEGFR2 and either HA-tagged WT epsin 1 or epsin 1 with Q9A, A13S, and K16A point mutations ( $n=4)$. (I) Western blot analysis of VEGFR2 co-IP by epsin 1 in 293 T cells overexpressing epsin 1 and either WT VEGFR2 or VEGFR2 with R1027A and R1080A point mutations $(n=4)$. (J) UPI peptide inhibition of epsin 1 IP by VEGFR2 KD (His) analyzed by coincubating control, UPI, or UPI-mutant peptides and anti-His Ab in lysates from $293 \mathrm{~T}$ cells overexpressing HA-tagged epsin 1 and His-tagged VEGFR2 KD $(n=5)$. Mut, mutant.

either HUVEC ${ }^{\alpha v \beta 3}$ or tumors (Supplemental Figure 3, J and K), nor did it disrupt VEGFR1 or Notch signaling in HUVEC ${ }^{\text {avp3 }}$ (Supplemental Figure 4). Notably, no VEGFR3 expression was detected in HUVEC $^{\text {avp3 }}$ (Supplemental Figure 4A).
It is well established that the epsin UIM binds ubiquitinated cell surface receptors, such as activated VEGFR2 $(17,18)$, partially via its highly conserved, albeit weak and nonspecific, interaction with ubiquitin (Figure 1D). However, the molecular mech- 

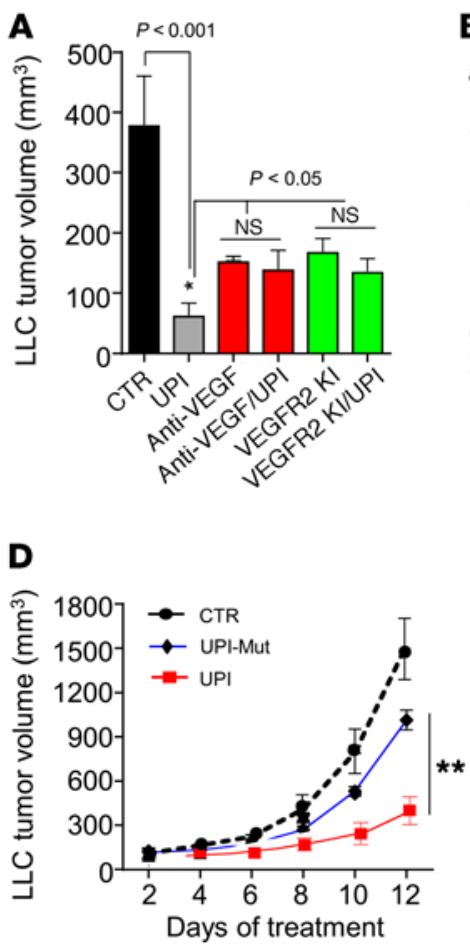
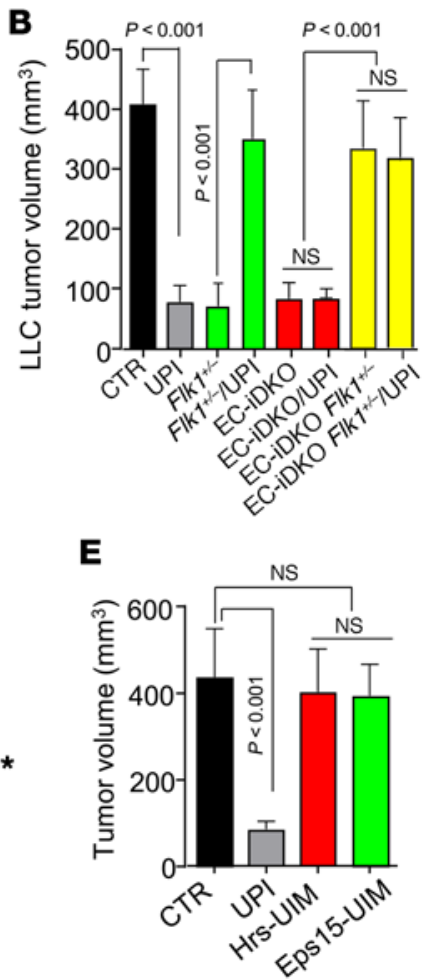

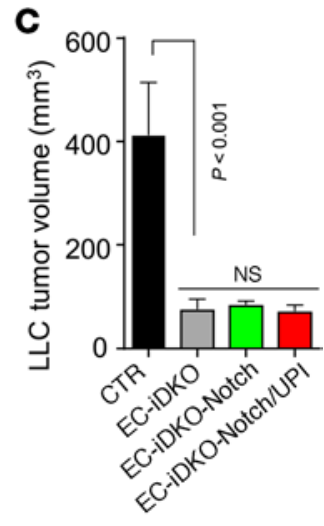

Figure 5. UPI mimetic specifically targets VEGFR2 signaling in genetically modified mouse models. (A) Tumor volumes from s.c. LLC tumor-bearing mice treated i.v. as indicated with control, UP peptide $(10 \mathrm{mg} / \mathrm{kg})$, anti-VEGF Ab $(1.5$ $\mathrm{mg} / \mathrm{kg}$ ), or VEGFR2 kinase inhibitor (KI) $(0.5 \mathrm{mg} / \mathrm{kg})(n=5) .{ }^{*} P<0.001$ and ${ }^{\#} P<0.05$, by 2 -tailed Student's $t$ test. (B and C) Tumor volumes from s.c. LLC tumor-bearing WT (B and C), WT Flk $\mathrm{T}^{+/-}$ (B), EC-iDKO (B and C), EC-iDKO Flk1+/(B), and EC-iDKO-Notch (C) mice treated i.v. with control or UPI peptide $(10 \mathrm{mg} / \mathrm{kg})$ $(n=5) .{ }^{*} P<0.001$, by 2 -tailed Student's $t$ test. (D) Tumor volumes from s.c. LLC tumor-bearing mice i.v. treated with control, UPI peptide, or UPI-Mut peptides $(10 \mathrm{mg} / \mathrm{kg})(n>5) .{ }^{* *} P<0.001$, by 2 -tailed Student's $t$ test and Bonferroni's multiple comparisons test. (E) Tumor volumes from s.c. LLC tumor-bearing mice treated i.v. with control, UPI peptide, Hrs-UIM, or Eps15-UIM peptides (10 mg/kg) $(n>5)$. ${ }^{*} P<0.001$, by 2 -tailed Student's $t$ test. anisms driving the specificity with which the epsin UIM or the UPI peptide interact with VEGFR2, but not with other ubiquitinated angiogenic receptors, in endothelial cells remain unknown. Molecular modeling predicted that, in addition to interacting with ubiquitinated VEGFR2, the UPI peptide docks directly into a previously unidentified pocket in the VEGFR2 KD (Figure 1E). The UIM residues predicted to mediate this docking are unique to the epsin UIM $(\mathrm{Q} 9, \mathrm{~A} 13$, and K16) and may play a critical role in mediating the specific interaction with residues R1027 and R1080 of VEGFR2 (Figure 4, E and F). Therefore, we speculated that $\mathrm{Q} 9, \mathrm{~A} 13$, and $\mathrm{K} 16$ in the epsin UIM possess specific biological functions for the regulation of epsin-VEGFR2 interactions and tumor angiogenesis. To definitively determine the importance of these residues in controlling epsin-binding affinity for VEGFR2, surface plasmon resonance (SPR) was used to analyze the binding affinity between recombinant VEGFR2 KD and WT or mutant UPI peptide containing Q9A, A13S, and K16A point mutations within the UIM (UPI-Mut) (Supplemental Table 1). UPI-Mut peptide markedly reduced the binding affinity for VEGFR2 KD compared with WT UPI peptide (Figure 4G). To further delineate the individual importance of these mutations, we generated a series of point mutations within these critical epsin UIM and VEGFR2 residues and used co-IP with anti-VEGFR2 or anti-epsin 1 to evaluate their effects on epsin 1-VEGFR2 binding. Predictably, Q9AA13S-K16A triple mutations in the epsin UIM attenuated epsin 1-VEGFR2 interactions in 293T cells (Figure 4H). Reciprocally, R1027A and R1080A mutations in VEGFR2 also decreased epsin 1-VEGFR2 interactions (Figure 4I). In further support of these data, the UPI-Mut peptide failed to disrupt epsin-VEGFR2 interaction when administered to 293T cells, indicating reduced binding of UPI-Mut peptide to VEGFR2 and failed competitive inhibi- tion of epsin (Figure 4J). Collectively, these data strongly support our molecular model predictions of a UPI peptide-binding pocket within VEGFR2 that provides the specificity with which the UPI peptide modulates VEGFR2 function.

UPI peptide selectively disrupts epsin-VEGFR2 interaction and proper VEGFR2 signaling regulation in genetically modified animal models. To interrogate UPI peptide as a specific modulator of VEGFR2 signaling in vivo, s.c. LLC tumor-bearing mice were treated with UPI peptide, anti-VEGF Ab, VEGFR2 kinase inhibitor, or a combination of UPI and anti-VEGF Ab or UPI and VEGFR2 kinase inhibitor. Consistent with earlier in vitro data (Supplemental Figure 3, A and B), coinjection of UPI peptide with either antiVEGF Ab or VEGFR2 kinase inhibitor impaired UPI peptide therapeutic efficacy (Figure 5A), further suggesting that UPI peptide specifically targets activated VEGFR2.

To further establish UPI peptide as a competitive inhibitor of epsin function as it pertains to VEGFR2 regulation in vivo, several unique animal models were used to establish s.c. LLC tumor models, including inducible endothelial cell-specific

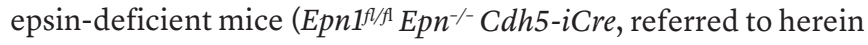
as EC-iDKO mice), WT mice, or EC-iDKO mice heterozygous for VEGFR2 ( $F l k 1^{+/-}$and EC-iDKO-Flk1 ${ }^{+/}$, respectively), and EC-iDKO mice that express a transgene encoding an active Notch, NICD, to rescue defective Notch signaling in EC-iDKO mice (EC-iDKO Nicd ${ }^{L S L}$, referred to herein as EC-iDKO-Notch mice) (Supplemental Figure 5A). Loss of a single VEGFR2 allele in WT mice $\left(F l k 1^{+/}\right)$significantly hindered VEGF signaling and, thereby, tumor growth compared with that seen in WT mice (Figure 5B and Supplemental Figure 5B). Administration of UPI peptide rescued the retarded tumor growth phenotype of $F l k 1^{+/-}$ mice (Figure 5B), because the UPI peptide acts as an inhibitor of 

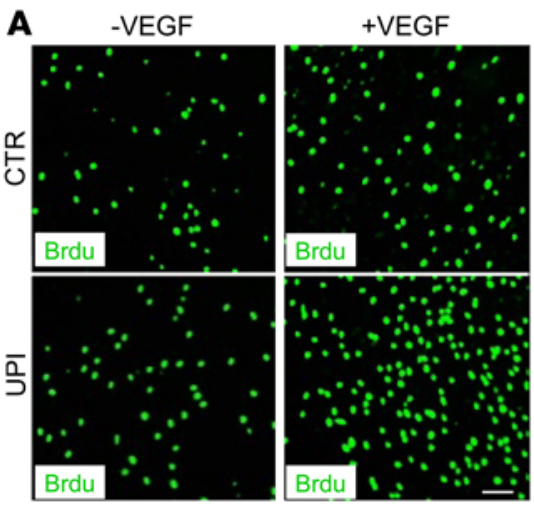

B

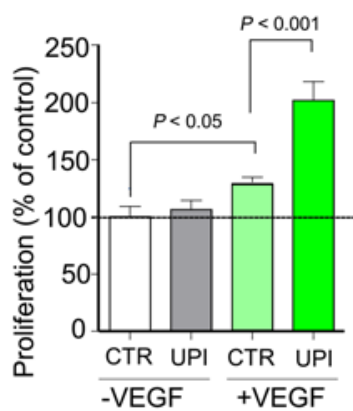

G

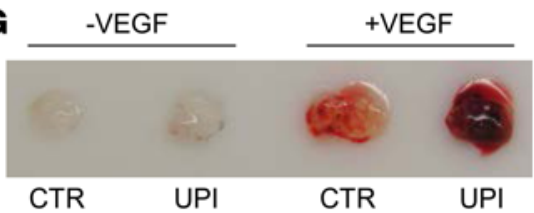

C

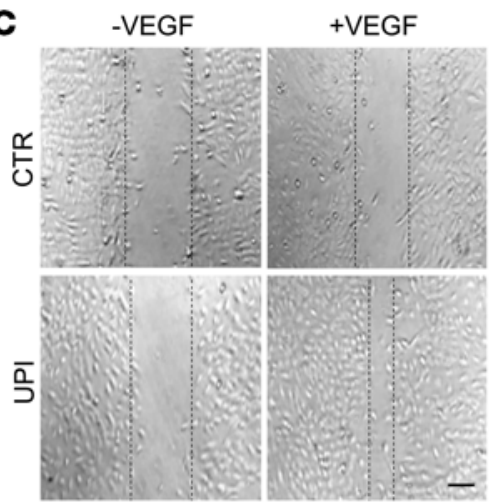

D

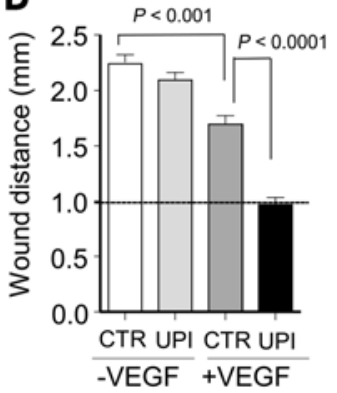

E

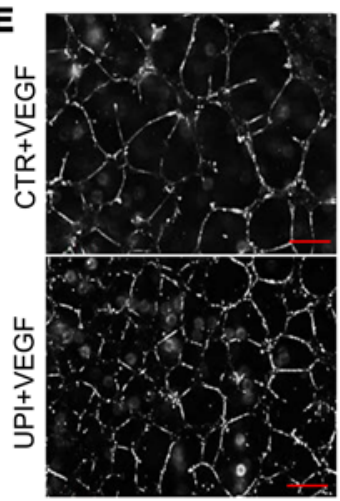

$\mathbf{F}$

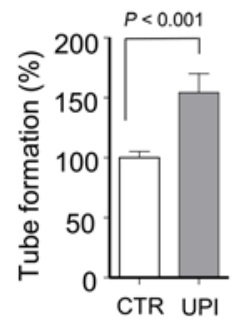

H

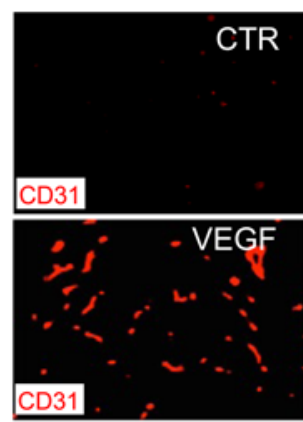

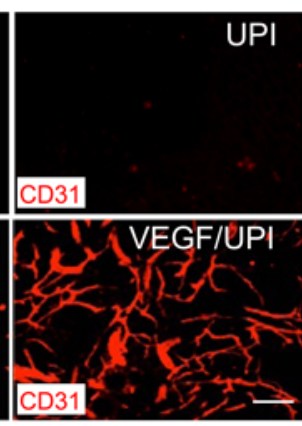

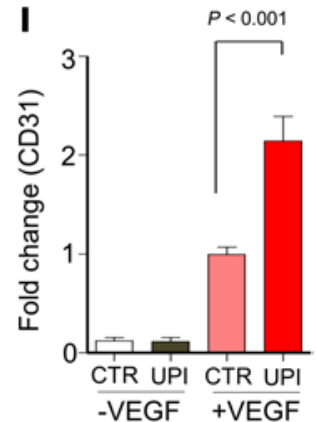

J

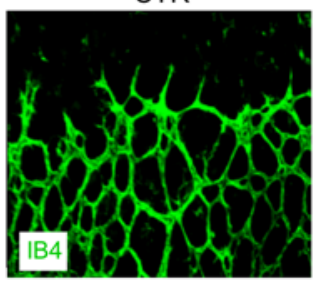

UPI

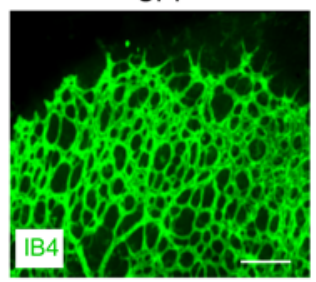

K

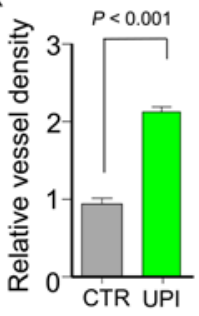

Figure 6. UPI mimetic promotes angiogenesis in vitro and in vivo. Representative image (A) and analysis (B) of VEGF-stimulated BrdU incorporation (proliferation) in control or UPI peptide-treated HUVEC ${ }^{\alpha u \beta 3}(n=5) . P<0.05$ and $P<0.001$, by 2-tailed Student's $t$ test. Scale bar: $100 \mu \mathrm{m}$. Representative image (C) and analysis (D) of VEGF-stimulated wound healing (migration) in control or UPI peptide-treated HUVEC avß3 $(n=5)$. $P<0.001$ and $P<0.0001$, by 2-tailed Student's $t$ test. Scale bar: $200 \mu \mathrm{m}$. Representative image (E) and analysis (F) of VEGF-stimulated Matrigel tube formation in control or UPI peptide-treated $\operatorname{HUVEC}^{\alpha \nu \beta 3}(n=4) . P<0.001$, by 2-tailed Student's $t$ test. Scale bar: $500 \mu \mathrm{m}$. (G-I) In vivo angiogenesis of VEGF-A and UPI peptide-containing Matrigel plugs (C). Isolated Matrigel plugs were harvested and processed for immunofluorescence staining with anti-CD31 Ab (H). Quantification of CD31 fluorescence intensity is shown in I $(n=5)$. Control versus UPI, $P<0.001$, by 2-tailed Student's $t$ test. Scale bar: $100 \mu \mathrm{m}$. (J and $\mathbf{K})$ Retinal neoangiogenesis of P6 pups treated intraocularly with control or UPI peptide was analyzed by immunofluorescence staining for the vascular marker isolectin B4 (IB4) ( $n=5)$. $P<0.001$, by 2 -tailed Student's $t$ test. Scale bar: $100 \mu \mathrm{m}$.

VEGFR2 internalization and degradation, resulting in sustained VEGFR2 signaling and normalized tumor angiogenesis (Supplemental Figure 5B). In contrast, UPI peptide treatment of either EC-iDKO or EC-iDKO $\mathrm{Flk1}^{+/-}$mice, in which epsins are specifi- cally depleted in endothelial cells, did not alter tumor volumes compared with control peptide (Figure 5B), implying that UPI peptide modulates tumor growth via its effect on epsin-VEGFR2 interaction (Supplemental Figure 5C); since epsins are depleted, 
UPI peptide is no longer effective. Further, no difference in tumor growth between EC-iDKO mice and EC-iDKO-Notch mice was seen upon UPI peptide treatment, suggesting that UPI peptide does not affect tumor angiogenesis through the Notch pathway (Figure 5C). By using these genetically modified animal models in combination with pharmacological studies, we concluded that UPI peptide hinders tumor growth by specifically inhibiting epsin-VEGFR2 binding, resulting in elevated VEGFR2 signaling and aberrant tumor angiogenesis.

UPI-Mut peptide administered to s.c. LLC tumor-bearing mice failed to inhibit tumor growth compared with that seen in mice treated with UPI peptide (Figure 5D). Given that the mutated residues (Q9A, A13A, and K16A) are unique to the epsin UIM, we wanted to determine their significance in facilitating epsin-VEGFR2 binding. To do this, we replaced the epsin UIM in the UPI peptide with UIMs from other endocytic proteins including Eps15 and Hrs, creating the peptides Eps15-UIM and Hrs-UIM (Supplemental Table 1). Importantly, when administered to LLC tumor-bearing mice, neither Eps15-UIM nor HrsUIM peptides affected tumor growth (Figure 5E). These results were similar to those obtained using the UPI-Mut peptide and in contrast with the effective tumor inhibition promoted by the UPI peptide, thus emphasizing the unique qualities within the epsin UIM that enable it to specifically interact with VEGFR2 to modulate VEGF signaling and tumor angiogenesis.

UPI peptide promotes in vitro and in vivo neoangiogenesis and disturbs functional tumor angiogenesis by stabilizing VEGFR2 signaling and prompting the formation of hyperleaky tumor vasculature. Given that the UPI peptide impaired downregulation of VEGFR2 signaling, we reasoned that it would promote VEGF-induced angiogenesis in in vitro angiogenesis assays. Control and UPI peptides were used to treat HUVEC ${ }^{a v \beta 3}$ in the presence or absence of VEGF. UPI peptide treatment greatly elevated VEGF-induced HUVEC ${ }^{\alpha v \beta 3}$ proliferation as determined by BrdU labeling (Figure 6, A and B), migration using wound-healing assays (Figure 6, C and D), and tube formation in a 3D Matrigel assay (Figure 6, E and F).

To complement the above in vitro angiogenic assays, we examined the effect of the UPI peptide on regulating neoangiogenesis using 2 standard models for in vivo neoangiogenesis: in vivo Matrigel plugs and retinal neovascularization. Matrigel, premixed with UPI or control peptide $(100 \mu \mathrm{g} / \mathrm{ml})$ in the presence or absence of VEGF, was implanted s.c. into C57BL/6 mice. Six days after implantation, Matrigel plugs were removed and processed for staining using the vascular endothelial marker CD31. The UPI peptide promoted VEGF-stimulated in vivo neoangiogenesis, as evidenced by increased new blood vessel formation in the UPI peptide-containing VEGF-positive Matrigel plugs relative to UPI peptide alone or control peptide plus VEGF (Figure 6, G-I). Similarly, intraocular injection of UPI peptide $(100 \mu \mathrm{g} / \mathrm{ml})$ into mouse pups at P1, P3, and P5 caused a significant increase in retinal neoangiogenesis, as determined by isolectin $\mathrm{B} 4$ immunofluorescence staining of retinae isolated at P6 (Figure 6, J and K). These findings strongly support our hypothesis that UPI peptide is a proangiogenic agent that promotes both in vitro and in vivo neoangiogenesis. Furthermore, these findings suggest that the UPI-targeting strategy is not limited to TECs, but applies also to endothelial cells that are activated and express high levels of $\alpha v \beta 3$ integrin.
In contrast, established vessels in adult mice were quiescent and expressed lower levels of $\alpha v \beta 3$ integrin. Thus, we predicted that UPI would have a minimal effect on normal vessels but an enormous impact on tumor vessels.

Of significance, upon examination of tumor vessels from control and UPI peptide-treated s.c. LLC and U87 tumors (by CD31 staining), UPI peptide induced tumor vessel proliferation and promoted their dilation (Figure 7, A-C). This result was further confirmed by immunostaining using anti-VE-cadherin Ab (Supplemental Figure 6). These aberrant vessel characteristics (indicative of aberrant tumor angiogenesis) are consistent with previous studies testing the effects of genetic epsin depletion on tumor angiogenesis (17). Further, VEGFR2 protein levels were dramatically increased in s.c. tumor models treated with UPI peptide (Figure 7D and Supplemental Figure 7A), indicating impaired VEGFR2 degradation. To further support this notion, we isolated TECs from control- or UPI peptide-treated LLC tumor-bearing mice and determined via Western blotting that UPI peptide treatment drastically increased both total and phosphorylated VEGFR2 (p-VEGFR2) (Figure 7E). VEGFR2 phosphorylation, as determined by immunofluorescence staining of s.c. U87 tumor vasculature, was also significantly augmented by UPI peptide injection (Figure $7 \mathrm{~F}$ and Supplemental Figure 7B). These findings further validate that UPI peptides specifically targets the VEGFR2 signaling pathway.

Administration of UPI peptide produced a striking morphological change in tumor vessels; therefore, we examined the effects of this change on tumor vessel function. Subcutaneous U87 tumor-bearing mice were treated with control or UPI peptide for 3 weeks, followed by perfusion with fixable FITC-dextran of high molecular weight. Mice were sacrificed and tumor vessels stained for CD31. UPI, but not control peptide, produced extensive leakage of the fluorescent dextran into tumor tissues (Figure 7G and Supplemental Figure 7C), indicative of vessel hyperpermeability, which coincided with decreased $\alpha$-smooth muscle actin ( $\alpha$-SMA) coverage of tumor vessels (Figure $7 \mathrm{H}$ and Supplemental Figure 7D). Examination by transmission electron microscopy (TEM) of s.c. tumors extracted from U87 tumor-bearing SCID mice revealed increased fenestrations and open cell-cell junctions between tumor vessel endothelial cells in UPI peptide-treated tumors (Supplemental Figure 7G). Consequently, semi-thin sections showed prominent rbc leakage from tumor vessels, accompanied by dying tumor cells in response to UPI peptide treatment (Figure 7I and Supplemental Figure 7G). Additionally, tumors from UPI peptide-treated mice showed increased hypoxia (Figure 7J and Supplemental Figure $7 \mathrm{E})$, presumably a result of impaired vascular delivery of oxygen. Notably, the vascular effects of UPI peptide are limited to tumor endothelium, with negligible hyperpermeability or leakage occurring in the quiescent endothelium, which expresses lower levels of $\alpha v \beta 3$ integrin, including inactive vasculatures in tissues like intestine (Figure 7K and Supplemental Figure 7F), brain, kidney, stomach, or liver (Supplemental Figure 7H). These data further support our results from the plasma biomarker examination (Supplemental Figure 7I), suggesting minimal toxicity of the peptide. Together, these data strongly suggest that UPI peptide treatment perturbs tumor vessel function specifically and results in profound leakage and damaging hypoxia. 
A

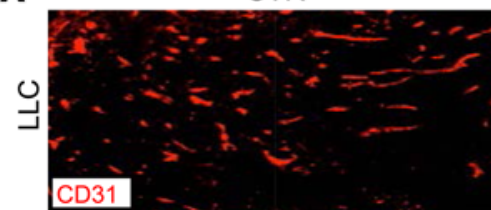

ڤิ

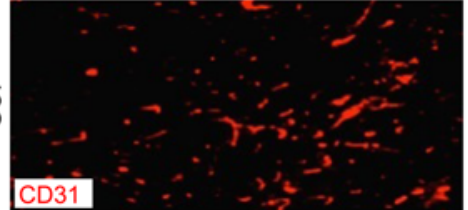

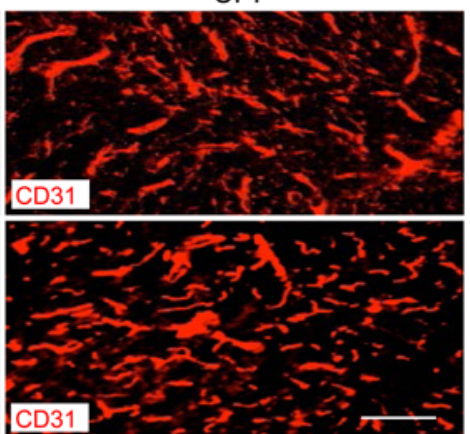

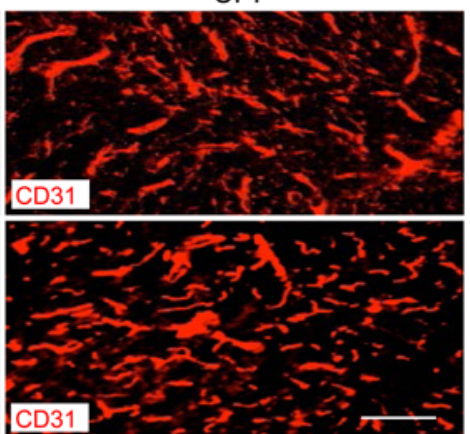

B

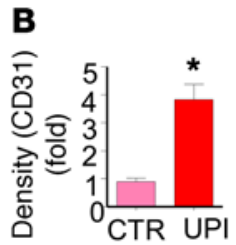

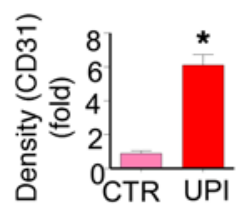

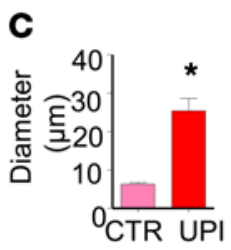

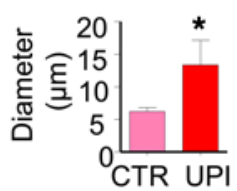

D

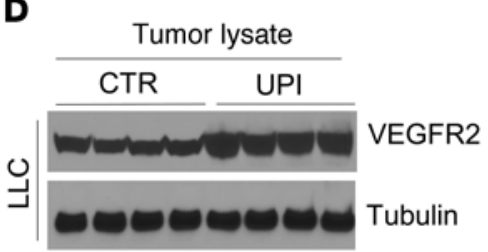

o

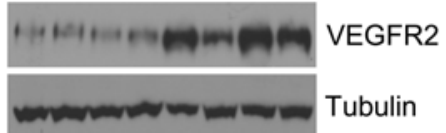

E

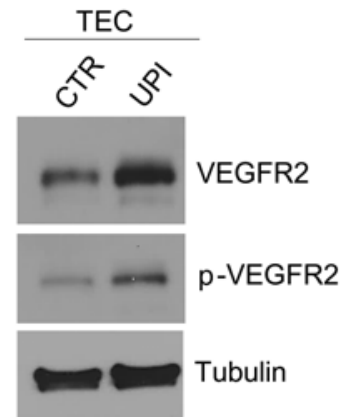

G

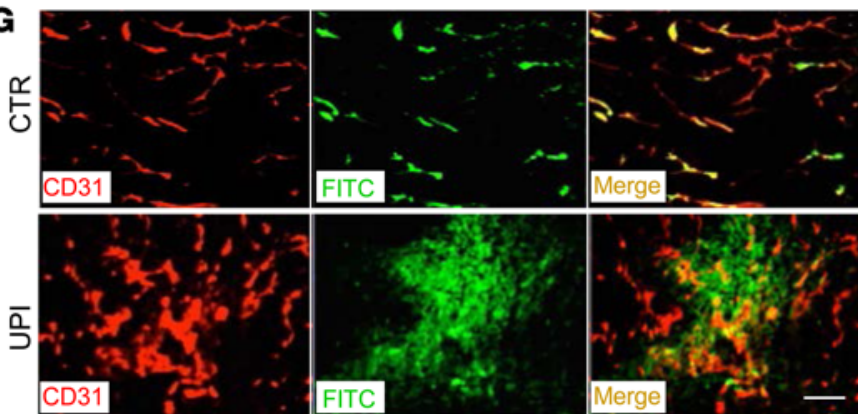

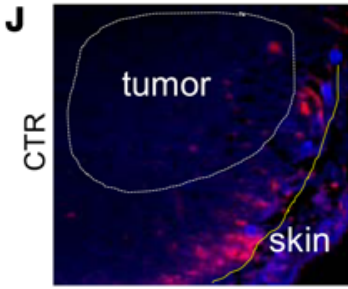

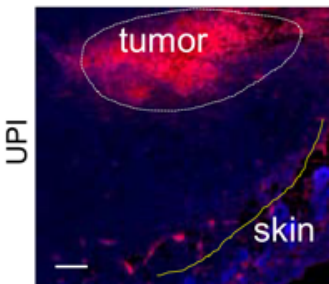

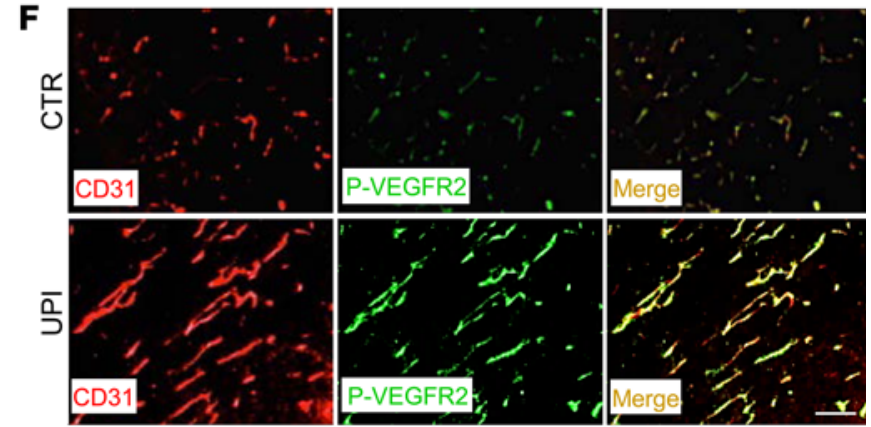

H

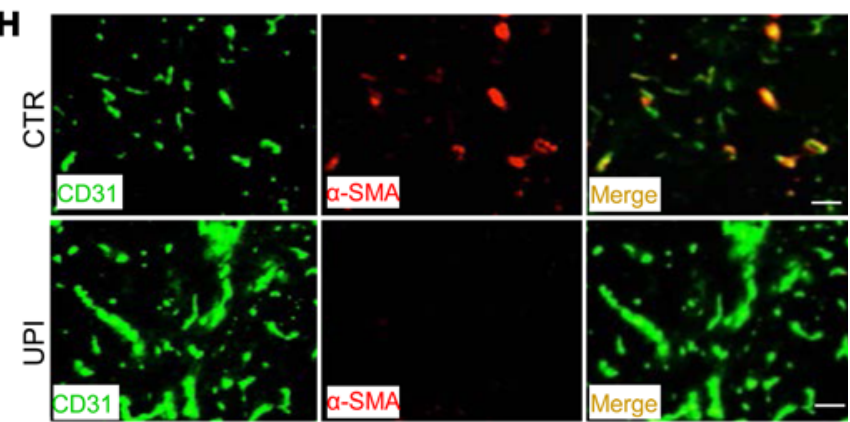

$\mathbf{K}$

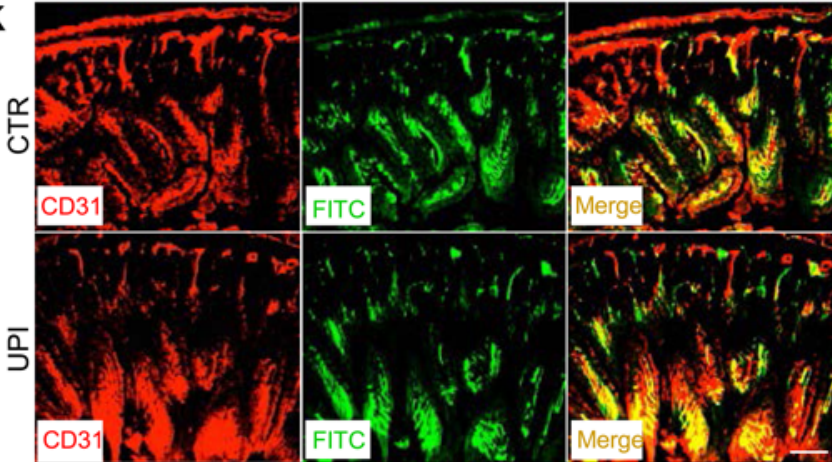


Figure 7. UPI mimetic promotes aberrant tumor angiogenesis. (A) CD31 immunofluorescence staining of control or UPI peptide-treated s.c. implanted LLC and U87 tumors. Scale bar: $100 \mu \mathrm{m}$. Vessel density (B) and diameter (C) were quantified ( $n=6$ tumors). ${ }^{*} P<0.001$, by 2 -tailed Student's $t$ test. (D) VEGFR2 expression in tumor lysates from control and UPI peptide-treated s.c. LLC and U87 tumors $(n=5)$. See also Supplemental Figure 6A for quantification. (E) Total and phosphorylated VEGFR2 in TECs isolated from control or UPI peptide-treated s.c. LLC tumor-bearing mice $(n=3)$. (F) CD31 and phosphorylated VEGFR2 immunofluorescence staining of control and UPI peptide-treated s.c. implanted U87 tumors $(n=6)$. Scale bar: $100 \mu \mathrm{m}$. See also Supplemental Figure 6B for quantification of p-VEGFR2 fluorescence intensity. (G) Control or UPI peptide-treated s.c. U87 tumor-bearing mice were perfused with FITC-dextran for 10 minutes, and then tumors were harvested and fixed for CD31 staining $(n=5)$. Scale bar: $100 \mu \mathrm{m}$. See also Supplemental Figure 6C for quantification of FITC leakage. (H) CD31 and $\alpha$-SMA immunofluorescence staining of control or UPI peptidetreated s.c. implanted U87 tumors $(n=6)$. Scale bar: $100 \mu \mathrm{m}$. See also Supplemental Figure 6D for quantification of $\alpha$-SMA coverage. (I) TEM analysis of semi-thin sections from control and UPI peptide-treated s.C. implanted U87 tumors. Dotted red lines indicate tumor vessels; blue arrows depict rbc leakage from tumor vessels; red arrows indicate dying tumor cells $(n=5)$. Scale bar: $50 \mu \mathrm{m}$. (J) Hypoxia probe pimonidazole hydrochloride immunofluorescence staining of control and UPI peptidetreated s.c. implanted U87 tumors $(n=6)$. Scale bar: $100 \mu \mathrm{m}$. See also Supplemental Figure 6 E for quantification. (K) Control and UPI peptidetreated s.c. U87 tumor-bearing mice were perfused with FITC-dextran for 10 minutes, and then major organs were harvested and fixed for CD31 staining $(n=5)$. Scale bar: $100 \mu \mathrm{m}$. See also Supplemental Figure $6 \mathrm{H}$ for representative images of brain, kidney, stomach, and liver.

UPI peptide treatment retards tumor growth in orthotopic glioblastoma models. To determine the preclinical implications of UPI peptide treatment as a potential human cancer therapeutic, we used mouse and human orthotopic glioma models. We began by orthotopically injecting GL261 mouse glioma cells into the forebrains of C57BL/6 mice. When tumor size reached 10-20 $\mathrm{mm}^{3}$, mice were randomized into 2 groups receiving either control or UPI peptide $(10 \mathrm{mg} / \mathrm{kg})$ by i.v. injection every other day. Tumor growth was monitored by MRI on alternating days. UPI peptide treatment hindered tumor growth with an efficacy similar to that of current anti-VEGF Ab therapy (Figure 8A). MRI analysis of tumor growth on day 23 after inoculation revealed considerable tumor size reductions in the UPI peptide-treated mice (Figure 8B). Remarkably, UPI peptide treatment also prolonged survival of tumor-bearing mice beyond the study endpoint, with an efficacy also comparable to that seen with antiVEGF Ab therapy (Figure $8 \mathrm{C}$ ). To investigate the potential for application of the UPI peptide in a human cancer model, we orthotopically implanted human glioma U87 cells into the right forebrain of immune-deficient SCID mice and subjected them to i.v. administration of control or UPI peptide $(10 \mathrm{mg} / \mathrm{kg})$ every 2 days, beginning after tumors were established $\left(10-20 \mathrm{~mm}^{3}\right)$. Tumor growth was monitored as described above. Consistently, UPI peptide therapy depressed U87 glioblastoma tumor growth (Figure 8, D and E). Impaired tumor growth after UPI peptide administration extended life expectancy of the orthotopic U87 glioma tumor-bearing SCID mice (Figure 8F). Although significant, the extended life expectancy of UPI peptide-treated human U87 glioma tumor-bearing SCID mice was not as dramatic as the increased life expectancy observed in GL261 glioma tumor-bear- ing C57BL/6 mice receiving UPI peptide therapy (Figure 8C). Given the importance of a functional immune system in combating tumor development and progression (37), our results offer concrete support for the synergistic effect of the UPI peptide and host immune defense in fighting tumor invasion. Strikingly, when U87 tumors of equal size were compared, MRI analysis revealed severe necrosis in the U87 glioma of UPI peptide-treated SCID mice, as evidenced by the appearance of large voids (Figure 8, G and $\mathrm{H}$ ). Notably, tumors receiving UPI peptide required almost twice as much time to achieve a tumor mass that was similar to that of control peptide-treated tumors (35 days compared with 18 days). Elevated necrosis after UPI peptide administration corresponded tightly with heightened VEGFR2 in the glioblastoma tumor vasculature, which was indicated by in situ labeling analysis of vascular VEGFR2 (Figure 7G and Figure 8I). These data support our earlier conclusions that UPI peptide hinders tumor growth by exacerbating VEGFR2-mediated tumor angiogenesis. Further, the corresponding increase in necrosis strongly suggests that the dysfunctional and hyperleaky tumor vessels resulting from UPI peptide treatment promote tumor cell death and lead to impaired tumor growth.

Coadministration of UPI peptide with cytotoxic chemotherapeutics further sustains tumor growth inhibition. UPI peptide treatment caused striking tumor vascular characteristics including vessel dilation, hyperleakage, and hyperplasticity (Figure 7). We postulate that the enhanced vascularization may increase the total number of cancer cells directly exposed to circulating blood and that this, in combination with the increased vascular permeabilization, may facilitate enhanced cytotoxic chemotherapeutic delivery. To test this, we combined the cytotoxic chemotherapeutic doxorubicin (Dox), Taxol (Tax), or OKN-007 (38-40) with UPI peptide administration to s.c. LLC tumor-bearing mice. Our results demonstrate that combined UPI/Dox or UPI/Tax further sustained tumor inhibition relative to single treatment with UPI, Dox, or Tax, or combined treatment with anti-VEGF/Dox or antiVEGF/Tax in the LLC tumor model (Figure 9A and Supplemental Figure 8), suggesting that the aberrant and hyperleaky tumor vessels resulting from UPI treatment may facilitate delivery of the cytotoxic chemotherapeutic to the surrounding tumor cells, which could be more effective than vessel normalization by anti-VEGF treatment. In accordance with enhanced cytotoxic chemotherapeutic delivery, we identified more apoptotic cells in the UPI peptide combinational groups relative to the anti-VEGF Ab combinational groups by TUNEL staining (Figure 9B and Supplemental Figure $8 \mathrm{~B}$ ). Similar results were also obtained in the orthotopic GL261 glioma tumor-bearing mouse model (Supplemental Figure 8, C and D). Collectively, our results demonstrate that UPI peptide more effectively hinders tumor growth relative to anti-VEGF Ab when combined with cytotoxic chemotherapeutics.

In summary, our data suggest that UPI peptide is a potent suppressive agent of tumor growth and metastasis that functions by disrupting tumor angiogenesis. Our data support this in various preclinical cancer models, including the human U87 glioblastoma model. We also show that administration of the UPI peptide can significantly increase survival rates and extend life expectancy, while imposing negligible toxicity. Coadministration of UPI with cytotoxic chemotherapeutics further increases therapeutic efficacy. 
A

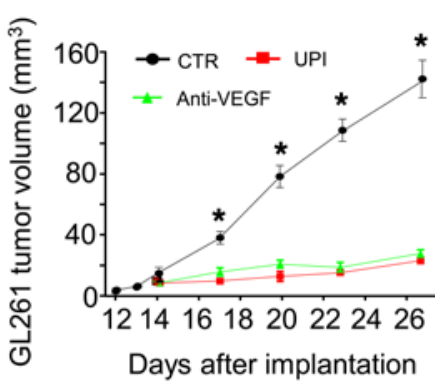

D

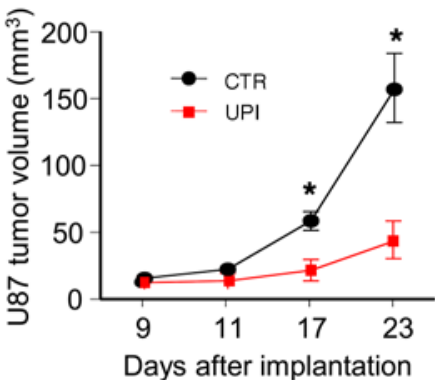

B

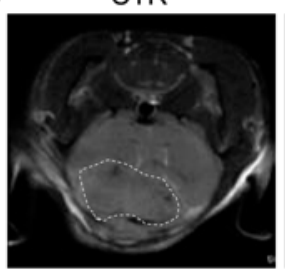

E

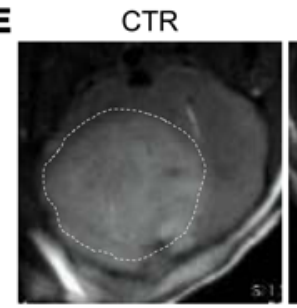

H

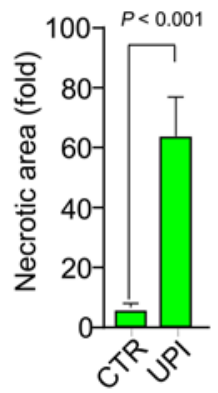

C

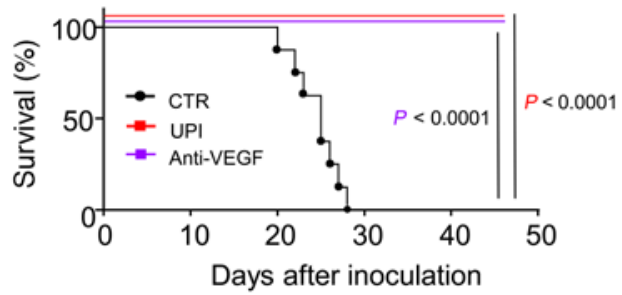

$\mathbf{F}$

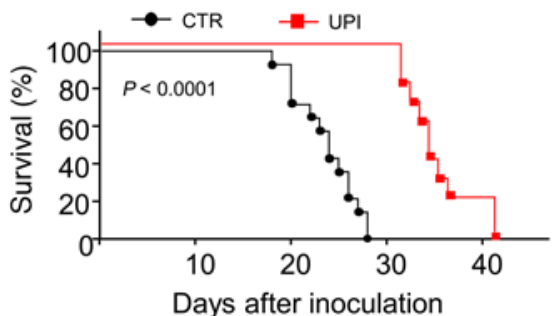

G

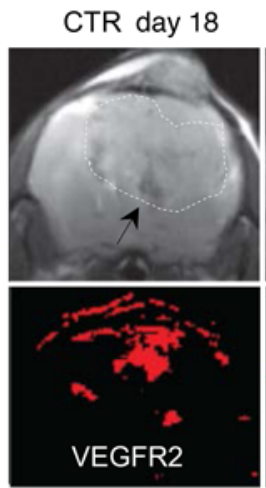

UPI day 35

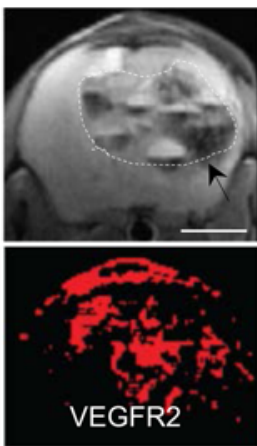

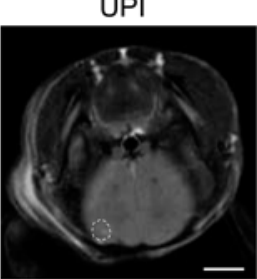

UPI

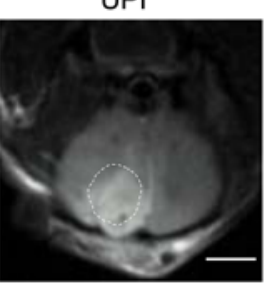

I

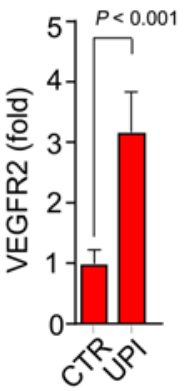

Figure 8. UPI mimetic suppresses tumor growth and promotes survival rate in orthotopic glioma mouse models. (A and B) Tumor volumes (analyzed by MRI) from orthotopically implanted GL261 glioblastoma tumor-bearing mice treated i.v. with control or UPI peptide (10 mg/kg, $n=10)$ every other day. Anti-VEGF Ab $(5 \mathrm{mg} / \mathrm{kg})$ served as a positive control $(n=4)$. White dotted lines indicate tumor in representative MRI images taken on day 23 after implantation (B) $(n=10)$. (A) ${ }^{*} P<0.001$, by 2-tailed Student's $t$ test and Bonferroni's multiple comparisons test. Scale bar: $3 \mathrm{~mm}$. (C) Survival plot of orthotopically implanted GL261 glioblastoma tumor-bearing mice injected i.v. with control or UPI peptide (10 mg $/ \mathrm{kg})$ every other day $(n=10) . P<0.0001$ by Mantel-Cox log-rank test. Anti-VEGF Ab $(5 \mathrm{mg} / \mathrm{kg})$ served as a positive control $(n=4) . P<0.0001$. (D and $\mathbf{E})$ Tumor volumes (analyzed by MRI) from orthotopically implanted human U87 glioblastoma tumor-bearing SCID mice treated i.v. with control or UPI peptide (10 mg/kg) $(n=10)$ every other day. White dotted lines indicate tumor in representative MRI images of terminal mice $(\mathbf{E})(n=10)$. (D) ${ }^{*} P<0.05$, by 2-tailed Student's $t$ test and Bonferroni's multiple comparisons test. Scale bar: $3 \mathrm{~mm}$. (F) Survival plot of orthotopically implanted human U87 glioblastoma tumor-bearing SCID mice injected i.V. with control or UPI peptide $(10 \mathrm{mg} / \mathrm{kg}$ ) every other day $(n=10) . P<0.0001$, by Mantel-Cox log-rank test. (G-I) MRI graphs of control or UPI peptide-treated orthotopically implanted human U87 glioblastoma tumor-bearing SCID mice with similar tumor sizes (day 18 [CTR] vs. day 35 [UPI]). White dotted lines and arrows indicate tumors; lower panels show in situ VEGFR2-tracking analysis. Green and red bars in $\mathbf{H}$ and $\mathbf{I}$ are quantifications of necrotic areas $(\mathbf{H})$ and VEGFR2 (I) in glioblastoma $(n=9)$. ( $\mathbf{H}$ and $\mathbf{I}) P<0.001$, by 2-tailed Student's $t$ test. Scale bar: $3 \mathrm{~mm}$.

\section{Discussion}

Tumor angiogenesis is a critical event in cancer progression and metastasis, making it an attractive target for anticancer therapeutic strategies $(3,13,41-45)$. Previous therapeutic approaches have focused predominantly on disrupting tumor angiogenesis by inhibiting proangiogenic signaling pathways such as VEGF and Notch. Alternatively, we propose a potentially fruitful new therapeutic strategy for suppressing tumor growth based on our previous discovery that loss of epsin-mediated VEGFR2 degradation also hinders tumor progression by impairing proper downregulation of VEGFR2 signaling and promoting subsequent uncontrolled tumor angiogenesis $(17,18)$. We further discovered that the epsin UIM played a critical regulatory role in VEGFR2 internalization and downregulation (17), thereby providing us with a strong therapeutic rationale to disrupt the interaction between epsin and VEGFR2 through competitive inhibition by an epsin UIM peptide (Figure 9C). Here, we report that an originally designed epsin mimetic UPI peptide produces remarkable inhibition of tumor growth in LLC (lung), B16 (melanoma), TRAMP (prostate), and U87 and GL261 (glioblastoma) preclinical cancer models (Figures 2 and 8). Similar to genetic endothelial cell-specific epsin depletion, our UPI peptide substantially stabilized VEGFR2 protein to enhance 

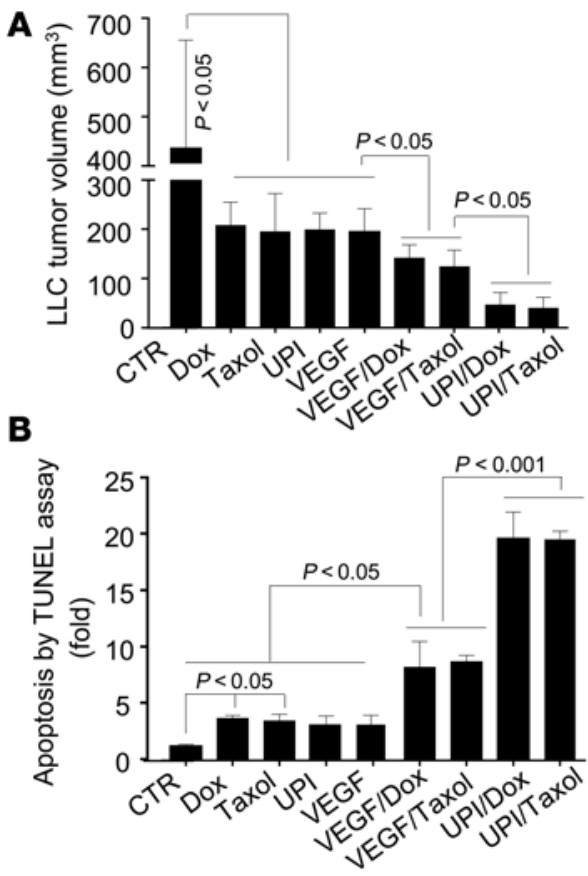

C

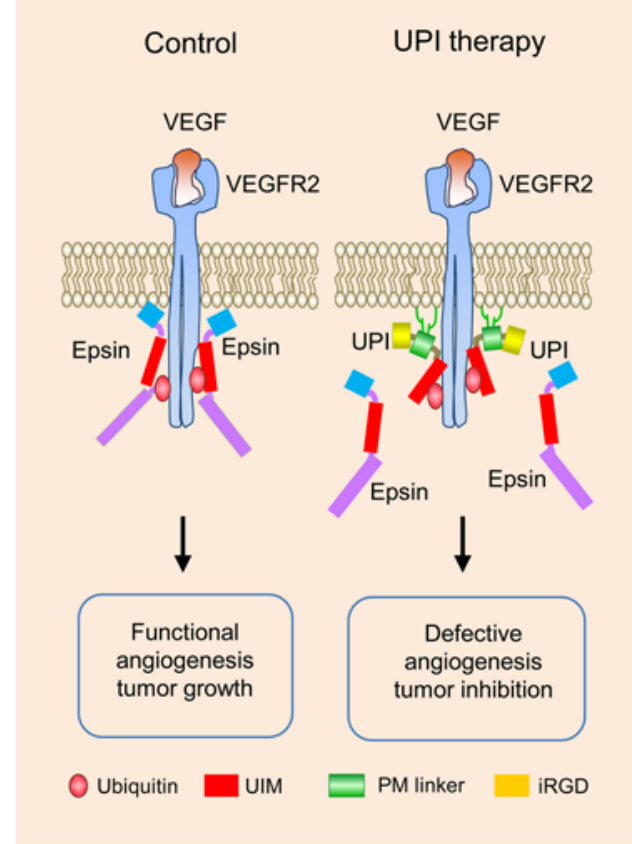

Figure 9. UPI peptide further sustains tumor inhibition in combinational therapy with chemotherapeutics. (A) Combinational therapy of UPI ( $8 \mathrm{mg} /$ $\mathrm{kg}$ ) with the cytotoxic chemotherapeutic Dox $(10 \mathrm{mg} / \mathrm{kg})$ or Tax $(5 \mathrm{mg} /$ $\mathrm{kg})$ in s.c. LLC tumor-bearing mice. Anti-VEGF Ab (VEGF) therapy $(2.5 \mathrm{mg} /$ $\mathrm{kg}$ ) combined with Dox or Tax was performed in parallel ( $n>5$ tumors). $P<0.05$. (B) TUNEL staining of LLC tumors from Supplemental Figure 8B $(n=5)$. (A and B) $P<0.05$ and $P<$ 0.001 , by 2-tailed Student's $t$ test and Bonferroni's multiple comparisons test. (C) Molecular mechanisms proposed for the underlying therapeutic action of UPI. UPI competes with endogenous epsin to interfere with epsin-mediated downregulation of activated VEGFR2 in tumor endothelium, thus disrupting tumor angiogenesis and leading to retarded tumor growth.
VEGFR2 signaling and produce disorganized, dilated, and dysfunctional leaky tumor vessels (Figures 4 and 7). Consequently, this peptide not only retarded tumor growth but also impeded cancer metastasis (Figures 2 and 3), thus providing a strong rationale for its potential as a new translational cancer therapeutic.

Therapeutic efficacy is dependent on several aspects including localized delivery, precise target specificity, and minimal toxicity. To optimize the therapeutic efficacy of an epsin UIM peptide (21), we incorporated a cyclic iRGD homing sequence (27) to ensure localized delivery to activated endothelial cells such as those in tumor vasculature and a plasma membrane-anchoring sequence (29) to precisely concentrate it within close proximity to activated VEGFR2 at the plasma membrane (Supplemental Figure 1D). In support of this strategy, our UPI peptide required a relatively low dose for effective tumor inhibition (Figure 2A) and exhibited negligible penetration of nontumor quiescent vasculature or tissues (Figure 1I). The fact that an every-other-day delivery approach was sufficient to impede tumor growth further suggests that these targeting sequences and the epsin UIM ensure targeted delivery and potent efficacy. Moreover, this strategy contributed significantly to the minimal toxicities observed (Supplemental Figure 7I).

The fact that UPI peptide treatment effectively and specifically disrupted epsin-VEGFR2 interaction, resulting in sustained VEGFR2 signaling, aberrant angiogenesis, and impaired tumor growth, demonstrates that the UPI peptide is an ideal mimetic of our previously described antitumor phenotype in endothelial-specific, epsin-deficient mice (17). From these findings, we concluded that the epsin UIM within our UPI peptide specifically targets VEGFR2, thereby competitively inhibiting endogenous epsin function and mirroring the tumor-inhibitory phenotype in epsin-deficient animals. This specificity was determined through the use of pharmacological agents, genetically modified animal models, biochemical approaches, and molecular modeling. As a result of these studies, we have identified a unique binding mechanism that governs the specificity with which the epsin UIM interacts with activated VEGFR2. The resulting interaction competitively prohibits epsin binding to VEGFR2 (Figure 1J) and subsequent epsin-mediated VEGFR2 internalization and downregulation (Supplemental Figure 1, G-I). Residues facilitating this interaction are unique to the epsin UIM and drive the specificity with which UPI peptide targets VEGFR2, but not other angiogenic receptors (Figure 4 ).

Given that neuropilin 1 is a reported coreceptor of VEGFR2 (46), it is possible that accumulation of VEGFR2 at the plasma membrane may hinder neuropilin 1-mediated UPI internalization by blocking the neuropilin 1 endocytosis necessary for UPI internalization. However, neuropilin 1 is reported to interact with (46) and facilitate VEGF165a-mediated VEGFR2 signaling propagation by directing VEGFR2 into the Rab5/Rab4/Rab11 recycling pathway (presumably via EEA1) $(47,48)$. In contrast, VEGF165b is a non-neuropilin 1-binding isoform of VEGF reported to direct VEGFR2 toward Rab7-dependent degradation (47). Given that UPI concentrates VEGFR2 on the plasma membrane by impeding the VEGF-targeted degradation pathway and that neuropilin 1 reportedly associates with VEGFR2 specifically in the endosomes (48), it is not likely that UPI-mediated VEGFR2 accumulation on the plasma membrane will affect neuropilin 1 internalization and/or recycling at the plasma membrane. In support of this, we observed dose-dependent and time-dependent increased, rather than decreased, UPI peptide accumulation (Supplemental Figure 1, B and C).

It is still debatable whether VEGFR2 signaling initiated at the plasma membrane differs from that originated at the early endosomes. Endocytosis and subsequent trafficking to lysosomes was traditionally thought to be a primary mechanism for extinguishing receptor signaling after ligand-induced dimerization and activation of a given RTK. VEGFR2, a prototype of such RTKs, undergoes similar downregulation by endocytosis (17). However, recent 
studies have also implicated VEGFR2 endocytosis and trafficking as an important regulatory mechanism promoting VEGFR2 signaling (48). Therefore, it is plausible that the destination of VEGFR2 for signaling endosomes or degradative lysosomes is regulated by different endocytic adaptors and that UPI peptide treatment specifically blocks VEGFR2 destined for degradation while promoting VEGFR2 rapid recycling via signaling endosomes back to the plasma membrane. This could lead to increased cell surface localization of VEGFR2 and elevated VEGFR2 signaling originating from the plasma membrane, signaling endosomes, or both (17, 49-51).

In glioma animal models, UPI peptide was equally as effective at inhibiting tumor growth as currently available anti-VEGF therapies (Figure 8, A and C). Clinically, leaky vessels could be devastating to patients with glioblastoma, because these vessels cause buildup of fluid, which exacerbates tissue damage by increasing intracranial pressure. However, we did not observe increased edema in the T2-weighted images from our glioma mouse models, nor did these mice exhibit phenotypes consistent with increased intracranial pressure. In contrast, mice treated with UPI peptide had much longer survival rates and decreased tumor volumes compared with those of control mice. Potential differences in responses to leaky vessels and the resulting intracranial pressure could be a disparity between preclinical mouse models and human patients. However, given the potential long-term benefits of UPI peptide treatment suggested by our preclinical studies, it may prove clinically beneficial to use shunts to relieve intracranial pressure, if it occurs.

Clinically, angiogenesis inhibitors such as anti-VEGF are often combined with cytotoxic chemotherapeutics to enhance efficacy. However, whether promoting, rather than inhibiting, aberrant tumor angiogenesis provides a more effective cytotoxic chemotherapeutic delivery strategy is unknown. Herein, we demonstrated that UPI peptide treatment significantly increased the efficacy of combined cytotoxic chemotherapeutics, including Dox, Tax, and OKN-007. Furthermore, this enhanced efficacy was substantially better relative to anti-VEGF combinatorial therapeutic approaches (Figure 9, A and B, and Supplemental Figure 8). While the mechanism is unclear, we hypothesize that the tumor vascular leakage induced by UPI peptide treatment facilitates the release of cytotoxic chemotherapeutics, thereby increasing cancer cell death (Figure 9, A and B, and Supplemental Figure 8). Simultaneously, increased vessel numbers in UPI peptide-treated tumors further encourages cancer cell exposure to the cytotoxic chemotherapeutics. It is conceivable that anti-VEGF therapy, by nature, reduces angiogenesis, and even with vessel normalization within the tumor, it may still result in less effective delivery of combinational cytotoxic chemotherapeutics relative to UPI peptide treatment. Additionally, the smaller molecular size, intracellular delivery, and cost-effective nature of peptide therapeutics make them a feasible and somewhat more preferable therapeutic strategy relative to $\mathrm{Ab}$ therapies, which are limited to extracellular or secretory targets and much more expensive to produce. In summary, when combined with cytotoxic chemotherapeutics, UPI peptide could offer more effective and sustained tumor inhibition clinically.

Although counterintuitive and opposing in function to other antiangiogenic approaches, our findings using the UPI peptide strongly suggest that the antitumorigenic effect of dysfunctional angiogenesis is a result of an altered VEGFR2 signaling balance and can be achieved by either inhibiting or facilitating signaling. Furthermore, our studies suggest that facilitating aberrant VEGFR2 signaling and the subsequent disruption in tumor vasculature can impair the growth of a variety of tumors, despite their heterogeneity in tumor endothelial VEGFR2 expression. However, the dramatic inhibitory and relatively long-term effects of UPI peptide on the characteristically high levels of VEGFR2 expression in glioma tumor endothelium suggest that UPI peptide therapies would likely be most potent when used to treat highly vascularized tumors with elevated endothelial VEGFR2 expression levels. Such findings would suggest that tumor heterogeneity might play a regulatory role in the efficacy of UPI targeting. Collectively, our originally designed localized tumor-targeting approach has yielded a UPI peptide that holds what we believe to be uniquely high antiangiogenic and antimetastatic potential for cancer treatment $(52,53)$.

\section{Methods}

\section{Peptide synthesis}

Peptides were synthesized by solid-phase fluorenylmethyloxycarbonyl (FMOC). The crude product was dissolved, separated, and confirmed by mass spectrometry, as described in the Supplemental Methods. After confirmation, peptides were purified by HPLC and lyophilized. Peptide endotoxin (EU/mg) was monitored using a Pierce LAL Chromogenic Endotoxin Quantitation Kit according to the manufacturer's instructions.

\section{Animal models}

All animal protocols were approved by the IACUC of the Oklahoma Medical Research Foundation. Flk1+-, EC-iDKO, EC-iDKO Flk1+/, and EC-iDKO-Notch mice were generated by crossing conditional double-KO mice (EC-iDKO) with Flk1 $1^{f / f l}$ or Nicd ${ }^{L S L}$ mice, as described in Supplemental Figure 5A and refs. 17 and 18. Subcutaneous (LLC, melanoma B16, and glioma U87/SCID), TRAMP, and orthotopic (GL261 and U87/SCID) animal tumor models were established and analyzed, as previously described $(17,18,54,55)$. Synthetic peptides were administered i.v. at $10 \mathrm{mg} / \mathrm{kg}$ or i.p. at $20 \mathrm{mg} / \mathrm{kg}$ every other day. Anti-VEGF $\mathrm{Ab}$ (B20-4.1.1; $1.5 \mathrm{mg} / \mathrm{kg})$ or VEGFR2 kinase inhibitor $(0.5 \mathrm{mg} / \mathrm{kg})$ were iv. coadministered with synthetic peptides.

\section{Kinetic analysis and FUPI peptide distribution in vivo}

Tumor and nontumor tissues from FUPI-treated LLC or B16 tumorbearing mice were collected and analyzed by immunofluorescence staining for FUPI (FITC) localization to CD31-positive vessels.

\section{In vivo angiogenesis assays}

In vivo angiogenesis was analyzed using Matrigel plugs and retinal neovascularization, as previously described $(18,51,56,57)$, with modifications.

\section{Hypoxia analysis in s.c. U87 glioma tumors}

Hypoxia probe was administered by i.p. injection into s.c. U87 glioma tumor-bearing mice. Tumor hypoxia was analyzed by immunofluorescence, as described in the Supplemental Methods. 
In situ VEGFR2 monitoring in the orthotopic U87 glioma model

VEGFR2-targeted MRI probe was administered to orthotopic U87 glioma tumor-bearing mice and analyzed, as previously described (58).

\section{Biochemical pulldown assay using ex vivo tumors}

Biotinylated synthetic peptides were used for biochemical pulldowns in isolated LLC tumors, as described in the Supplemental Methods.

\section{Construction and mammalian expression of human VEGFR2 KD}

Human VEGFR2 KD (59) was cloned and inserted into the mammalian expression vector pcDNA3, as described in the Supplemental Methods.

\section{Construction and purification of recombinant VEGFR2 KD protein}

The BacPAK Baculovirus Expression System (Clontech) was used to purify VEGFR2 KD, as previously reported (59-61).

\section{SPR analysis of UPI-VEGFR2-binding affinity}

Binding of UPI or UPI-Mut (Q9A, A13S, and K16A) peptides to VEGFR2 KD was analyzed by SPR using a Biacore 2000 biosensor (GE Healthcare).

\section{UIM peptide competition (epsin 1-VEGFR2 interference) assay} 293 T cells cotransfected with epsin 1-HA (tag) and VEGFR2 KD-His (tag) were lysed and subjected to IP using anti-His tag Ab (GenScript) in the presence of synthetic peptides.

UPI peptide binding to angiogenic receptors in HUVEC ${ }^{\alpha v \beta 3}$ by biotin-ELISA VEGF-stimulated HUVEC ${ }^{a v \beta 3}$ were lysed, then coincubated with biotinylated UPI or control peptides with anti-VEGFR2 Ab (or anti-PDGFR- $\beta$, anti-EGFR, or anti-FGFR1 Abs) and rec-G beads (Invitrogen) for IP. Immunocomplexes were eluted by low $\mathrm{pH}$, neutralized, and subjected to biotin-ELISA analysis.

Bioinformatic analysis of UPI peptides and putative binding with VECFR2 Molecular dynamics simulation. 3D structures of UIM and UPI were predicted by PEP-FOLD (http://bioserv.rpbs.univ-paris-diderot.fr/ PEP-FOLD) and analyzed, as previously described $(62,63)$.

Molecular docking procedure. Docking experiments were performed using the ClusPro program (http://cluspro.bu.edu/login.php) $(64,65)$.

\section{Statistics}

Data are presented as the mean \pm SEM. Data were analyzed by a 2 -tailed Student's $t$ test or ANOVA, with Bonferroni's test for multiple comparisons. A $P$ value of less than 0.05 was considered statistically significant.

\section{Study approval}

All animal studies were reviewed and approved by the IACUC of the OMRF.

Full details of the methods are provided in the Supplemental Methods.

\section{Author contributions}

YD and HC designed, analyzed, and interpreted the data. YD conducted most of the in vitro and animal experiments and handled the data presentation. HNAR performed statistical analysis. HW performed the molecular modeling work. HW, PMD, and RPM conducted the SPR-binding assay. SP performed the site mutagenesis for epsin 1 and VEGFR2 experiments. XL conducted the VEGFR1, VEGFR3, and Notch signaling experiments in vitro. JD handled the glioma tumor therapy. RSM and FL performed the EM experiments. DS, CN, and RT helped with MRI measurement of the glioma tumors. YL helped with the Ve-cadherin immune-fluorescence staining procedures. KS performed the in vitro peptide-binding assay. $\mathrm{XC}, \mathrm{BC}, \mathrm{MLB}$, and $\mathrm{HS}$ helped with critical discussions. LY, SH, and JM performed mouse breeding and genotyping and provided technical support. LX, RSS, and DB helped with animal models and discussions. KLT, YD, and HC wrote the manuscript.

\section{Acknowledgments}

Monoclonal anti-VEGF Ab was provided by Genentech Inc. We thank Jin Chen for insightful comments and helpful discussions. We thank Hua Zhu, Ruby Rahman, Melinda Lay, Yiyuan Chen, Alexis Chen, and Lingli Yu for their technical assistance and the OMRF Imaging Core for help with sample preparation. This work was supported in part by NIH grants R01HL-093242, R01 HL118676, and P20 RR018758; Oklahoma Center for Advanced Science and Technology (OCAST) grant HR09-116; American Heart Association (AHA) National Scientific Development grant 0835544N; Department of Defense grant W81XWH-11-1-00226 (to H. Chen); NIH grant P01HL085607 (to L. Xia and R.P. McEver); NIH grant SC1DK104821 (to Y. Liu); OCAST grants AR11-043 and HR14-056 and AHA-SDG grant 12SDG8760002 (to Y. Dong); AHA Postdoctoral Fellowship awards 13POST16940008 (to K.L. Tessneer), 13POST17270006 (to S. Pasula), and 15POST21310008 (to H. Song); AHA Predoctoral Fellowship awards RSRCH016952 (to X. Liu) and 15PRE21400010 (to M.L Brophy); NIH Postdoctoral Fellowship award 1 F32 HL121954-01A1 (to K.L. Tessneer); and NIH Predoctoral Fellowship award 1F31HL127982-01 (to M.L. Brophy). J. Dong was supported by the OMRF Fleming Scholar Program.

Address correspondence to: Yunzhou Dong or Hong Chen, Boston Children's Hospital, 300 Longwood Avenue, Boston, Massachusetts 02115, USA. Phone: 617.919.2435; E-mail: yunzhou.dong@ childrens.harvard.edu (Y. Dong). Phone: 617.919.6304; E-mail: hong.chen@childrens.harvard.edu (H. Chen).

Yunzhou Dong and Hong Chen's present address is: Vascular Biology Program and Department of Surgery, Boston Children's Hospital, Harvard Medical School, Boston, Massachusetts, USA.

\footnotetext{
1. Folkman J. Tumor angiogenesis: therapeutic implications. N EnglJMed.1971;285(21):1182-1186.

2. Dvorak HF. Vascular permeability factor/ vascular endothelial growth factor: a critical cytokine in tumor angiogenesis and a potential target for diagnosis and therapy. JClin Oncol. 2002;20(21):4368-4380.
}

3. Ferrara N, Kerbel RS. Angiogenesis as a therapeutic target. Nature. 2005;438(7070):967-974.

4. Kerbel RS. Tumor angiogenesis. N Engl J Med 2008;358(19):2039-2049.

5. Weis SM, Cheresh DA. Tumor angiogenesis: molecular pathways and therapeutic targets. Nat Med. 2011;17(11):1359-1370.
6. Carmeliet P, Jain RK. Molecular mechanisms and clinical applications of angiogenesis. Nature 2011;473(7347):298-307.

7. Ziyad S, Iruela-Arispe ML. Molecular mechanisms of tumor angiogenesis. Genes Cancer. 2011;2(12):1085-1096.

8. Gaur P, Bose D, Samuel S, Ellis LM. Targeting 
tumor angiogenesis. Semin Oncol. 2009; 36(2 suppl 1):S12-S19.

9. Kim KJ, et al. Inhibition of vascular endothelial growth factor-induced angiogenesis suppresses tumour growth in vivo. Nature. 1993;362(6423):841-844.

10. Brower V. Tumor angiogenesis - new drugs on the block. Nat Biotechnol. 1999;17(10):963-968.

11. Presta LG, et al. Humanization of an anti-vascular endothelial growth factor monoclonal antibody for the therapy of solid tumors and other disorders. Cancer Res. 1997;57(20):4593-4599.

12. Ferrara N, Hillan KJ, Novotny W. Bevacizumab (Avastin), a humanized anti-VEGF monoclonal antibody for cancer therapy. Biochem Biophys Res Commun. 2005;333(2):328-335.

13. Jain RK, Duda DG, Clark JW, Loeffler JS. Lessons from phase III clinical trials on anti-VEGF therapy for cancer. Oncology. 2006;3(1):24-40.

14. Ferrara N, Hillan KJ, Gerber HP, Novotny W. Discovery and development of bevacizumab, an anti-VEGF antibody for treating cancer. Drug Discov. 2004;3(5):391-400.

15. Jubb AM, Oates AJ, Holden S, Koeppen H. Predicting benefit from anti-angiogenic agents in malignancy. Nat Rev Cancer. 2006;6(8):626-635.

16. Bergers G, Hanahan D. Modes of resistance to anti-angiogenic therapy. Nat Rev Cancer. 2008;8(8):592-603.

17. Pasula S, et al. Endothelial epsin deficiency decreases tumor growth by enhancing VEGF signaling. J Clin Invest. 2012;122(12):4424-4438.

18. Tessneer KL, et al. Genetic reduction of vascular endothelial growth factor receptor 2 rescues aberrant angiogenesis caused by epsin deficiency. Arterioscler Thromb Vasc Biol. 2014;34(2):331-337.

19. Gould GW, Lippincott-Schwartz J. New roles for endosomes: from vesicular carriers to multi-purpose platforms. Nat Rev Mol Cell Biol. 2009;10(4):287-292.

20. Polo S, Pece S, Di Fiore PP. Endocytosis and cancer. Curr Opin Cell Biol. 2004;16(2):156-161.

21. Chen H, et al. Epsin is an EH-domain-binding protein implicated in clathrin-mediated endocytosis. Nature. 1998;394(6695):793-797.

22. Chen H, De Camilli P. The association of epsin with ubiquitinated cargo along the endocytic pathway is negatively regulated by its interaction with clathrin. Proc Natl Acad Sci U S A. 2005;102(8):2766-2771.

23. Messa M, et al. Epsin deficiency impairs endocytosis by stalling the actin-dependent invagination of endocytic clathrin-coated pits. Elife. 2014;3:e03311.

24. Coon BG, Direnzo DM, Konieczny SF, Aguilar RC. Epsins' novel role in cancer cell invasion. Commun Integr Biol. 2011;4(1):95-97.

25. Tessneer KL, et al. Epsin family of endocytic adaptor proteins as oncogenic regulators of cancer progression. JCan Res Updates. 2013;2(3):144-150.

26. Chen $\mathrm{H}$, et al. Embryonic arrest at midgestation and disruption of Notch signaling produced by the absence of both epsin 1 and epsin 2 in mice. Proc Natl Acad Sci U S A. 2009;106(33):13838-13843.

27. Sugahara KN, et al. Tissue-penetrating delivery of compounds and nanoparticles into tumors. Cancer Cell. 2009;16(6):510-520.
28. Sugahara KN, et al. Coadministration of a tumor-penetrating peptide enhances the efficacy of cancer drugs. Science. 2010;328(5981):1031-1035.

29. Kovarova M, Tolar P, Arudchandran R, Draberova L, Rivera J, Draber P. Structure-function analysis of Lyn kinase association with lipid rafts and initiation of early signaling events after Fcepsilon receptor I aggregation. Mol Cell Biol. 2001;21(24):8318-8328.

30. Swanson KA, Kang RS, Stamenova SD, Hicke L, Radhakrishnan I. Solution structure of Vps27 UIM-ubiquitin complex important for endosomal sorting and receptor downregulation. EMBO J. 2003;22(18):4597-4606.

31. Bogdanowich-Knipp SJ, Chakrabarti S, Williams TD, Dillman RK, Siahaan TJ. Solution stability of linear vs. J Pept Res. 1999;53(5):530-541.

32. Greenberg NM, et al. Prostate cancer in a transgenic mouse. Proc Natl Acad Sci US A. 1995;92(8):3439-3443.

33. Joliot A, Prochiantz A. Transduction peptides: from technology to physiology. Nat Cell Biol. 2004;6(3):189-196

34. Zetter BR. Angiogenesis and tumor metastasis. Annu Rev Med. 1998;49:407-424.

35. Harlozinska A. Progress in molecular mechanisms of tumor metastasis and angiogenesis. Anticancer Res. 2005;25(5):3327-3333.

36. Saharinen P, Eklund L, Pulkki K, Bono P, Alitalo $\mathrm{K}$. VEGF and angiopoietin signaling in tumor angiogenesis and metastasis. Trends Mol Med. 2011;17(7):347-362.

37. de Visser KE, Eichten A, Coussens LM. Paradoxical roles of the immune system during cancer development. Nat Rev Cancer. 2006;6(1):24-37.

38. Towner RA, et al. Regression of glioma tumor growth in F98 and U87 rat glioma models by the Nitrone OKN-007. Neuro Oncol. 2013;15(3):330-340.

39. de Souza PC, et al. OKN-007 decreases tumor necrosis and tumor cell proliferation and increases apoptosis in a preclinical F98 rat glioma model [published online ahead of print April 29, 2015]. JMagn Reson Imaging. doi:10.1002/jmri.24935.

40. He T, et al. Effects of PBN and OKNOO7 in roden glioma models assessed by $1 \mathrm{H}$ MR spectroscopy. Free Radic Biol Med. 2011;51(2):490-502.

41. Saaristo A, Karpanen T, Alitalo K. Mechanisms of angiogenesis and their use in the inhibition of tumor growth and metastasis. Oncogene. 2000;19(53):6122-6129.

42. Yancopoulos GD, Davis S, Gale NW, Rudge JS, Wiegand SJ, Holash J. Vascular-specific growth factors and blood vessel formation. Nature. 2000;407(6801):242-248.

43. Ridgway J, et al. Inhibition of Dll4 signalling inhibits tumour growth by deregulating angiogenesis. Nature. 2006;444(7122):1083-1087.

44. Noguera-Troise I, et al. Blockade of Dll4 inhibits tumour growth by promoting non-productive angiogenesis. Nature. 2006;444(7122):1032-1037.

45. Dikic I, Schmidt MH. Notch: Implications of endogenous inhibitors for therapy. Bioessays. 2010;32(6):481-487.

46. Prahst C, et al. Neuropilin-1-VEGFR-2 complexing requires the PDZ-binding domain of neuropilin-1. J Biol Chem. 2008;283(37):25110-25114.

47. Ballmer-Hofer K, Andersson AE, Ratcliffe LE,
Berger P. Neuropilin-1 promotes VEGFR-2 trafficking through Rab11 vesicles thereby specifying signal output. Blood. 2011;118(3):816-826.

48. Simons M. An inside view: VEGF receptor trafficking and signaling. Physiology. 2012;27(4):213-222.

49. Bonifacino JS, Traub LM. Signals for sorting of transmembrane proteins to endosomes and lysosomes. Annu Rev Biochem. 2003;72:395-447.

50. Traub LM. Sorting it out: AP-2 and alternate clathrin adaptors in endocytic cargo selection. JCell Biol. 2003;163(2):203-208.

51. Nakayama M, et al. Spatial regulation of VEGF receptor endocytosis in angiogenesis. Nat Cell Biol. 2013;15(3):249-260.

52. Burgess DJ. Angiogenesis: A happy medium? Nat Rev Cancer. 2013;13(1):4-5.

53. Klauber-Demore N. Are epsins a therapeutic target for tumor angiogenesis? J Clin Invest. 2012;122(12):4341-4343.

54. Tessneer KL, et al. Endocytic adaptor protein epsin is elevated in prostate cancer and required for cancer progression. ISRN Oncol. 2013;2013:420597.

55. Doblas S, et al. In vivo characterization of several rodent glioma models by $1 \mathrm{H}$ MRS. NMR Biomed. 2012;25(4):685-694.

56. Liu Z, et al. VEGF and inhibitors of TGF $\beta$ type-I receptor kinase synergistically promote blood-vessel formation by inducing alpha5-integrin expression. JCell Sci. 2009;122(pt 18):3294-3302.

57. Valapala M, Thamake SI, Vishwanatha JK. A competitive hexapeptide inhibitor of annexin A2 prevents hypoxia-induced angiogenic events. J Cell Sci. 2011;124(pt 9):1453-1464.

58. He T, et al. Molecular MRI differentiation of VEGF receptor-2 levels in C6 and RG2 glioma models. Am J Nucl Med Mol Imaging. 2013;3(4):300-311.

59. McTigue M, Murray BW, Chen JH, Deng YL, Solowiej J, Kania RS. Molecular conformations, interactions, and properties associated with drug efficiency and clinical performance among VEGFR TK inhibitors. Proc Natl Acad Sci U S A. 2012;109(45):18281-18289.

60. Leppanen VM, et al. Structural determinants of vascular endothelial growth factor-D receptor binding and specificity. Blood. 2011;117(5):1507-1515.

61. Leppanen VM, et al. Structural determinants of growth factor binding and specificity by VEGF receptor 2. Proc Natl Acad Sci U S A. 2010;107(6):2425-2430.

62. Maupetit J, Derreumaux P, Tuffery P. PEP-FOLD: an online resource for de novo peptide structure prediction. Nucleic Acids Res. 2009;37(Web server issue):W498-W503.

63. Thevenet P, Shen Y, Maupetit J, Guyon F, Derreumaux P, Tuffery P. PEP-FOLD: an updated de novo structure prediction server for both linear and disulfide bonded cyclic peptides. Nucleic Acids Res. 2012;40(Web server issue):W288-W293.

64. Comeau SR, Gatchell DW, Vajda S, Camacho CJ. ClusPro: a fully automated algorithm for protein-protein docking. Nucleic Acids Res. 2004;32(Web server issue):W96-W99.

65. Comeau SR, Gatchell DW, Vajda S, Camacho CJ ClusPro: an automated docking and discrimination method for the prediction of protein complexes. Bioinformatics. 2004;20(1):45-50. 